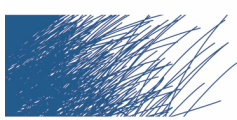

\title{
$\widehat{\overline{I I I I I I}}$ \\ UNIVERSITEIT \\ GENT
}

biblio.ugent.be

The UGent Institutional Repository is the electronic archiving and dissemination platform for all UGent research publications. Ghent University has implemented a mandate stipulating that all academic publications of UGent researchers should be deposited and archived in this repository. Except for items where current copyright restrictions apply, these papers are available in Open Access.

This item is the archived peer-reviewed author-version of:

Title: Tauberian theorems for the wavelet transform

Authors: Jasson Vindas, Stevan Pilipovic, Dusan Rakic

In: Journal of Fourier Analysis and Applications 17 (1), 65-95, 2011

To refer to or to cite this work, please use the citation to the published version:

J. Vindas, S. Pilipovic, D. Rakic (2011). Tauberian theorems for the wavelet transform. J. Fourier Anal. Appl. 17 (1), 65-95. doi: 10.1007/s00041-010-9146-1 


\title{
TAUBERIAN THEOREMS FOR THE WAVELET TRANSFORM
}

\author{
JASSON VINDAS, STEVAN PILIPOVIĆ AND DUŠAN RAKIĆ
}

\begin{abstract}
We make a complete wavelet analysis of asymptotic properties of distributions. The study is carried out via Abelian and Tauberian type results, connecting the boundary asymptotic behavior of the wavelet transform with local and non-local quasiasymptotic properties of elements in the Schwartz class of tempered distributions. Our Tauberian theorems are full characterizations of such asymptotic properties. We also provide precise wavelet characterizations of the asymptotic behavior of elements in the dual of the space of highly time-frequency localized functions over the real line. For the use of the wavelet transform in local analysis, we study the problem of extensions of distributions initially defined on $\mathbb{R} \backslash\{0\}$ to $\mathbb{R}$; in this extension problem, we explore the asymptotic properties of extensions of a distribution having a prescribed asymptotic behavior. Our results imply intrinsic properties of functions and measures as well, for example, we give a new proof of the classical Littlewood Tauberian theorem for power series.
\end{abstract}

\section{INTRODUCTION}

The aim of this paper is to provide a local analysis of distributions through the analysis of their wavelet transforms at boundary points. This will be done via a Tauberian approach. Our Abelian-Tauberian type results imply intrinsic properties of functions as well, for example, we will derive from them the nowhere differentiability of the Weierstrass function even in an average sense, Example 3, the celebrated Littlewood Tauberian theorem [25], Example 5, while in Examples 2 and 4 we consider Fourier series with gaps and in Example 6 we examine the asymptotics of monotone functions and non-negative measures via their wavelet transforms. Applications are also indicated in Remarks 1 and 2.

The wavelet transform is a powerful tool for studying local properties of functions. Usually, the wavelet analysis presents two main important features $[4,6,18,23,28,41]$ : the wavelet transform as a time-frequency analysis tool, and the wavelet analysis as part of approximation and function space

2000 Mathematics Subject Classification. 42C40, 26A12, 40E05, 41A60, 46F10, 42F12.

Key words and phrases. Wavelet transform; Abelian theorems; Tauberian theorems; inverse theorems; distributions; quasiasymptotics; slowly varying functions.

This work is supported by a Project of the Ministry of Science and Technological development of Serbia. J. Vindas gratefully acknowledges support by a Postdoctoral Fellowship of the Research Foundation-Flanders (FWO, Belgium). 
theory (see also $[4,16,17,27]$ and references therein for another approach to the time-frequency analysis). The existent applications of wavelet methods in local analysis are very rich. In [39], the wavelet transform is effectively applied to the analysis of differentiability properties of functions; it is deeply involved in the analysis of regularity notions. One could mention its vital role for the analysis of various classes of spaces, for example, ZygmundHölder type spaces and 2-microlocal spaces (cf. [19], [35], [36], [21]-[23], [28], [39]), and hence for the study of pseudodifferential operators within such classes (see $[3,18,20,23,28]$ ).

In this article, we are mainly concerned with the Schwartz class of tempered distributions. While it is totally clear what one means by the pointwise regularity and the asymptotic properties of a function, it is not so clear for a distribution. Schwartz definition of a distribution itself makes no allusion to pointwise properties, and, at a first look, suggests that one could hardly talk about them.

It is then interesting to mention that there are indeed local notions which can be used to measure pointwise properties of distributions. One may even talk about the value of a distribution at point, if one interprets it in the sense of Łojasiewicz [26]. Naturally, not all distributions have a value at a point. The Łojasiewicz notion admits a natural generalization, the quasiasymptotic behavior, which may be used to describe pointwise asymptotic properties of distributions as well as asymptotic properties at infinity. The quasiasymptotics were introduced by Zavialov [54] as a result of his investigations in quantum field theory, and further developed by him, Vladimirov and Drozhzhinov (see [7]-[10], [47]-[50]). Later on, the theory had its main developments within the study of integral transforms, convolution equations, partial differential equations, multiresolution expansions and Abelian and Tauberian theory (see [7]-[10], [13]-[15], [30]-[33], [38], [47]-[53]).

Ideas of quasiasymptotics have also important connections with problems in Fourier analysis, especially the case of Łojasiewicz notion for point values which has been widely used to study various problems of summability for Fourier series and integrals ([12], [43]-[45], [52]).

The main goal of this paper is to provide a complete study of the quasiasymptotic behavior of distributions through the wavelet transform which can be thought as a sort of mathematical microscope analyzing a distribution on various length scales around any point of the real axis. Therefore, this transform is very suitable for studying the quasiasymptotic behavior, which actually measures scaling self-similarities of distributions at an asymptotic level. We would like to point out that our results are related to those of Drozhzhinov and Zavialov, though with a different approach. In fact, the Tauberians from [8] make use of wavelets with finitely many vanishing moments, while here we employ wavelets with infinitely many vanishing moments.

The examples mentioned at the beginning of this introduction show that our analysis, connecting abstract notions from distribution theory with the 
wavelet transform, gives a powerful new tool for the local analysis of functions as well. Main structural theorems based on our results from previous papers are important and formulated in Theorems 1 and 2. Tauberian results are also given in the form of theorems; Theorems 3-7 are devoted to the behavior at a finite point while Theorems 8-11 are dedicated to the behavior at infinity. The Tauberian results for finite points relate the quasiasymptotics with asymptotics of the wavelet transform over cones with vertex at the boundary and the Tauberian estimate:

$$
\left|\mathcal{W}_{\psi} f\left(x_{0}+\varepsilon \cos \vartheta, \varepsilon \sin \vartheta\right)\right| \leq C \frac{\rho(\varepsilon)}{(\sin \vartheta)^{m}}
$$

for a suitable comparison function $\rho$. The unbounded term $(\sin \vartheta)^{-m}$ in (1.1) gives to the results a very general character with mild constrains.

The paper is organized as follows. We recall in Section 2 the basic facts from distribution wavelet analysis, following Holschneider [18]; we also briefly discuss the notion of quasiasymptotics. Section 3 connects the boundary asymptotic behavior of the wavelet transform through Abelian theorems and Tauberian characterizations of the quasiasymptotic behavior in $\mathcal{S}_{0}^{\prime}$, the dual of the space of highly time-frequency localized functions $\mathcal{S}_{0}$ [18]. In addition, we provide examples related to point values of distributions which correspond to Abelian type results and have some interesting consequences when applied to the Weierstrass function. We study in Section 4 the consequences of asymptotic relations in $\mathcal{S}_{0}^{\prime}$ within the space of tempered distributions. Moreover, we shall study in Section 4 a slightly more general problem, that is, the asymptotic properties of extensions to $\mathbb{R}$ of distributions initially defined on $\mathbb{R} \backslash\{0\}$ and having a prescribed asymptotic behavior; here we follow the approach from [42, 43], and complement some results. Notice that the latter is also important from a mathematical physics perspective, since it is of relevance for renormalization procedures in quantum field theory [2]. Sections 5 and 6 are the most important ones; there we obtain the Tauberian theorems for quasiasymptotics of tempered distributions in terms of the wavelet transform. These Tauberian theorems are complete inverse theorems to the Abelian ones from [32, 33]. They can also be considered as generalizations of the results from [19] to our distributional context. Within these sections, applications of the Tauberian results are given through examples and remarks. Finally, Appendix 7 contains the proof of a technical lemma from Section 3 (Lemma 1).

\section{Preliminaries and Notation}

The set of positive real numbers is denoted by $\mathbb{R}_{+}$and as usual its closure is denoted by $\overline{\mathbb{R}}_{+}=[0, \infty)$. Similarly, we use notation $\mathbb{R}_{-}$and $\overline{\mathbb{R}}_{-} ; \mathbb{R}_{0}=$ $\mathbb{R} \backslash\{0\}$. The sets of positive and negative integers are denoted by $\mathbb{Z}_{+}$and $\mathbb{Z}_{-} ; \mathbb{N}=\{0,1,2, \ldots\}$. The set $\mathbb{H}$ denotes the upper half-plane, that is, $\mathbb{H}=\mathbb{R} \times \mathbb{R}_{+}$. 
The Schwartz spaces of tests functions and distributions on the real line are denoted by $\mathcal{D}(\mathbb{R})$ and $\mathcal{D}^{\prime}(\mathbb{R})$, respectively; the spaces of smooth rapidly decreasing function and its dual, the space of tempered distributions, are denoted by $\mathcal{S}(\mathbb{R})$ and $\mathcal{S}^{\prime}(\mathbb{R})([34])$. The Fourier transform is defined by

$$
\mathcal{F}(\phi)(x)=\hat{\phi}(x)=\int_{-\infty}^{\infty} \phi(t) e^{-i x t} \mathrm{~d} t, \phi \in \mathcal{S}(\mathbb{R}),
$$

and extend to $\mathcal{S}^{\prime}(\mathbb{R})$ by duality. By a progressive distribution or function, we mean one whose Fourier transform is supported by $\overline{\mathbb{R}}_{+}$; similarly, the term regressive refers to those whose Fourier transform is supported by $\overline{\mathbb{R}}_{-}$.

We will follow [18] for the wavelet analysis of distributions. The space of highly time-frequency localized progressive functions over the real line $\mathcal{S}_{+}(\mathbb{R})$ is the set of those elements of $\mathcal{S}(\mathbb{R})$ which are progressive functions; correspondingly, $\mathcal{S}_{-}(\mathbb{R})$ consists of those ones which are regressive. The space $\mathcal{S}_{0}(\mathbb{R})$ is defined then as $\mathcal{S}_{0}(\mathbb{R})=\mathcal{S}_{-}(\mathbb{R}) \oplus \mathcal{S}_{+}(\mathbb{R})$ and it is called the space of highly time-frequency localized functions over $\mathbb{R}$. Alternatively, $\phi \in \mathcal{S}_{0}(\mathbb{R})$ if $\phi \in \mathcal{S}(\mathbb{R})$ and

$$
\int_{-\infty}^{\infty} x^{n} \phi(x) \mathrm{d} x=0, \text { for all } n \in \mathbb{N} .
$$

We note that $\mathcal{S}_{0}(\mathbb{R})$ is a closed subspace of $\mathcal{S}(\mathbb{R})$. The dual spaces of $\mathcal{S}_{+}(\mathbb{R})$, $\mathcal{S}_{-}(\mathbb{R})$ and $\mathcal{S}_{0}(\mathbb{R})$ (these spaces provided with the relative topology inhered from $\mathcal{S}(\mathbb{R}))$ are $\mathcal{S}_{-}^{\prime}(\mathbb{R})=\left(\mathcal{S}_{+}(\mathbb{R})\right)^{\prime}, \mathcal{S}_{+}^{\prime}(\mathbb{R})=\left(\mathcal{S}_{-}(\mathbb{R})\right)^{\prime}$ and $\mathcal{S}_{0}^{\prime}(\mathbb{R})$, respectively. It should be noticed that the space $\mathcal{S}_{+}^{\prime}(\mathbb{R})$ defined above is different from the one used in [47], for example.

Note that there is a continuous linear projector from $\mathcal{S}^{\prime}(\mathbb{R})$ onto $\mathcal{S}_{0}^{\prime}(\mathbb{R})$, given by the transpose of the trivial inclusion from $\mathcal{S}_{0}(\mathbb{R})$ to $\mathcal{S}(\mathbb{R})$. Due to the Hahn-Banach theorem, this map is surjective; however, there is no continuous right inverse for this projection [13]. Note also that the kernel of this projection is the space of polynomials; hence, the space $\mathcal{S}_{0}^{\prime}(\mathbb{R})$ can be regarded as the quotient space of $\mathcal{S}^{\prime}(\mathbb{R})$ by the space of polynomials. If $f \in \mathcal{S}^{\prime}(\mathbb{R})$, we will keep calling by $f$ the projection of $f$ to $\mathcal{S}_{0}^{\prime}(\mathbb{R})$.

By a wavelet we mean an element $\psi \in \mathcal{S}_{0}(\mathbb{R})$. The wavelet transform of $f \in \mathcal{S}^{\prime}(\mathbb{R})$ with respect to a wavelet $\psi$ is given by the $C^{\infty}$-function on $\mathbb{H}$

$$
\mathcal{W}_{\psi} f(b, a)=\langle f(b+a x), \bar{\psi}(x)\rangle=\left\langle f(t), \frac{1}{a} \bar{\psi}\left(\frac{t-b}{a}\right)\right\rangle=f * \check{\bar{\psi}}_{a}(b),
$$

where $\psi_{a}(\cdot)=\frac{1}{a} \psi(\dot{a})$.

The wavelet $\eta$ is called a reconstruction wavelet for the wavelet $\psi$ if the two constants

$$
c_{\psi, \eta}^{ \pm}=\int_{0}^{\infty} \overline{\hat{\psi}}( \pm x) \hat{\eta}( \pm x) \frac{\mathrm{d} x}{x}<\infty
$$

are non-zero and equal to each other; in such case we write

$$
c_{\psi, \eta}=c_{\psi, \eta}^{+}=c_{\psi, \eta}^{-}=\frac{1}{2} \int_{-\infty}^{\infty} \overline{\hat{\psi}}(x) \hat{\eta}(x) \frac{\mathrm{d} x}{|x|} .
$$


If $\psi$ admits the reconstruction wavelet $\eta$, we have the desingularization formula for $f \in \mathcal{S}_{0}^{\prime}(\mathbb{R})$ and $\phi \in \mathcal{S}_{0}(\mathbb{R})$

$$
\langle f(x), \phi(x)\rangle=\frac{1}{c_{\psi, \eta}} \int_{0}^{\infty} \int_{-\infty}^{\infty} \mathcal{W}_{\psi} f(b, a) \mathcal{W}_{\bar{\eta}} \phi(b, a) \frac{\mathrm{d} b \mathrm{~d} a}{a} .
$$

Therefore, the wavelet transform is injective on $\mathcal{S}_{0}^{\prime}(\mathbb{R})$; on the other hand, the injectivity of this integral transform fails when it is considered over $\mathcal{S}^{\prime}(\mathbb{R})$ because the moment vanishing condition (2.1) gives that the wavelet transform of any polynomial vanishes. Note that any wavelet admits a reconstruction wavelet as long as $\operatorname{supp} \hat{\psi} \cap \mathbb{R}_{+} \neq \emptyset$ and $\operatorname{supp} \hat{\psi} \cap \mathbb{R}_{-} \neq \emptyset$. We will mainly use wavelets admitting a reconstruction wavelet. An explicit example of one of such wavelets is $\psi$ given in the Fourier side by $\hat{\psi}(x)=$ $e^{-|x|-\frac{1}{|x|}}, x \in \mathbb{R}$, which is itself its own reconstruction wavelet.

We now change our attention to the concept of quasiasymptotic behavior of distributions $[15,30,42,43,46,47]$. The idea of quasiasymptotics is to look for asymptotic representations at either small scale or large scale. Specifically, we look for asymptotics

$$
f(\lambda x) \sim \rho(\lambda) g(x), \quad \text { as } \lambda \rightarrow 0^{+}, \text {or } \lambda \rightarrow \infty,
$$

in the distributional sense, that is, holding after evaluation at each test function

$$
\langle f(\lambda x), \phi(x)\rangle \sim \rho(\lambda)\langle g(x), \phi(x)\rangle, \quad \text { for each } \phi \in \mathcal{D}(\mathbb{R}) .
$$

If one assumes that $\rho$ is defined, positive and measurable near 0 (resp. $\infty$ ) and that $g$ is a non-zero distribution, then relation (2.4) forces $\rho$ to be a regularly varying function [1] and $g$ a homogeneous distribution having the degree of homogeneity equal to the index of regular variation of $\rho[15,30,47]$. Since any regularly varying function $\rho$ can be written as $\rho(h)=h^{\alpha} L(h)$, where $L$ is a slowly varying function, we may only talk about slowly varying functions in the rest of our discussion. Recall [1] a measurable real valued function defined and positive on an interval of the form $(0, A](\operatorname{resp} .[A, \infty))$, $A>0$, is called slowly varying at the origin (resp. at infinity) if

$$
\lim _{\varepsilon \rightarrow 0^{+}} \frac{L(a \varepsilon)}{L(\varepsilon)}=1, \text { resp. } \lim _{\lambda \rightarrow \infty} \frac{L(a \lambda)}{L(\lambda)}=1 .
$$

Observe that slowly varying functions are very convenient objects to be employed in wavelet analysis since they are asymptotically invariant under rescaling at small scale (resp. large scale). Recall,

Definition 1. A distribution $f \in \mathcal{D}^{\prime}(\mathbb{R})$ is said to have quasiasymptotic behavior of degree $\alpha$ at $x=x_{0}$ with respect to the slowly varying function $L$ if

$$
\lim _{\varepsilon \rightarrow 0^{+}} \frac{1}{\varepsilon^{\alpha} L(\varepsilon)}\left\langle f\left(x_{0}+\varepsilon x\right), \phi(x)\right\rangle
$$

exists (and is finite) for each $\phi \in \mathcal{D}(\mathbb{R})$. 
Note that the Banach-Steinhaus theorem implies that there must be a distribution $g \in \mathcal{D}^{\prime}(\mathbb{R})$ such that the above limit $(2.6)$ is equal to $\langle g(x), \phi(x)\rangle$, for each $\phi \in \mathcal{D}(\mathbb{R})$. As remarked before, if $g \neq 0$, then it must be a homogeneous distribution of degree $\alpha$. We recall that all homogeneous distributions on the real line are linear combinations of either $x_{+}^{\alpha}$ and $x_{-}^{\alpha}$, if $\alpha \notin \mathbb{Z}_{-}$, or $\delta^{(k-1)}(x)$ and $x^{-k}$, if $\alpha=-k \in \mathbb{Z}_{-}$. For these special distributions we are following the notation from [15]; other special distributions that we will use are $H(x)$, the Heaviside function, $\operatorname{sgn} x$, the signum function, and the pseudo-functions $\operatorname{Pf}\left(H( \pm x) / x^{k}\right), k \in \mathbb{Z}_{+}$.

Example 1. Point values of distributions. An important special case of Definition 1 is the value of distributions at a point in the sense of Łojasiewicz $[26,44]$, which is obtained when $\alpha=0$ and $L=1$. A distribution $f$ is said to have a (distributional) point value at $x_{0}$ in the sense of Łojasiewicz if

$$
\lim _{\varepsilon \rightarrow 0} f\left(x_{0}+\varepsilon x\right)=\gamma \quad \text { in } \mathcal{D}^{\prime}(\mathbb{R}) .
$$

In such a case we write $f\left(x_{0}\right)=\gamma$, distributionally. Note $([26,46])$, when $f \in L_{\mathrm{loc}}^{1}(\mathbb{R})$, the existence of $f\left(x_{0}\right)=\gamma$, distributionally, is equivalent to the existence of $m \in \mathbb{N}$ such that

$$
\lim _{x \rightarrow 0} \frac{m}{x} \int_{0}^{x} f\left(x_{0}+t\right)\left(1-\frac{t}{x}\right)^{m-1} \mathrm{~d} t=\gamma .
$$

Analogously to the quasiasymptotics at finite points, one defines the quasiasymptotics at infinity.

Definition 2. A distribution $f \in \mathcal{D}^{\prime}(\mathbb{R})$ has quasiasymptotic behavior of degree $\alpha$ at infinity in $\mathcal{D}^{\prime}(\mathbb{R})$ with respect to a slowly varying function $L$ if there exists $g \in \mathcal{D}^{\prime}(\mathbb{R})$ such that

$$
\lim _{\lambda \rightarrow \infty}\left\langle\frac{f(\lambda x)}{\lambda^{\alpha} L(\lambda)}, \phi(x)\right\rangle=\langle g(x), \phi(x)\rangle, \quad \forall \phi \in \mathcal{D}(\mathbb{R}) .
$$

Contrary to the case at points, the quasiasymptotic behavior at $\infty$ is not a local property, since any distribution of compact support satisfies the Estrada-Kanwal moment asymptotic expansion [15]:

$$
f(\lambda x) \sim \sum_{n=0}^{\infty} \frac{(-1)^{n} \mu_{n}}{n ! \lambda^{n+1}} \delta^{(n)}(x) \quad \text { as } \lambda \rightarrow \infty,
$$

where $\mu_{n}=\left\langle f(x), x^{n}\right\rangle$.

We may also talk about quasiasymptotics in other spaces of distributions, say $\mathcal{A}^{\prime}$ the dual of a suitable space of functions $\mathcal{A}$, meaning that $f \in \mathcal{A}^{\prime}$ and the test functions in (2.6), resp. (2.7), can be taken from $\mathcal{A}$. There is an obvious dependence on the space of generalized functions to be employed, so to denote the quasiasymptotics at infinity, we will indistinctly use the two convenient notations

$$
f(\lambda x) \sim \lambda^{\alpha} L(\lambda) g(x) \quad \text { as } \lambda \rightarrow \infty \text { in } \mathcal{A}^{\prime},
$$


and

$$
f(\lambda x)=\lambda^{\alpha} L(\lambda) g(x)+o\left(\lambda^{\alpha} L(\lambda)\right) \quad \text { as } \lambda \rightarrow \infty \text { in } \mathcal{A}^{\prime} .
$$

Likewise, an analogous notation will be used for quasiasymptotics at finite points.

In this article we are mainly interested in tempered distributions. It is very well known [46] that if $f \in \mathcal{S}^{\prime}(\mathbb{R})$ and it has quasiasymptotic behavior at a point in $\mathcal{D}^{\prime}(\mathbb{R})$, then it will have the same quasiasymptotic behavior in $\mathcal{S}^{\prime}(\mathbb{R})$ at the point. The same assertion holds for quasiasymptotics at infinity $[42,43,47]$.

\section{Characterization of Quasiasymptotics in $\mathcal{S}_{0}^{\prime}(\mathbb{R})$}

3.1. Abelian Type Results. Recently, the asymptotic behavior of the wavelet transform of a distribution having a quasiasymptotic behavior at a point has been investigated in $([32,33])$. Indeed, it is fairly easy to show that

$$
f\left(x_{0}+\varepsilon x\right) \sim \varepsilon^{\alpha} L(\varepsilon) g(x) \quad \text { as } \varepsilon \rightarrow 0^{+} \text {in } \mathcal{S}^{\prime}(\mathbb{R}),
$$

implies

$$
\mathcal{W}_{\psi} f\left(x_{0}, a\right) \sim L(a) \mathcal{W}_{\psi} g(0, a)=a^{\alpha} L(a) \mathcal{W}_{\psi} g(0,1), \quad a \rightarrow 0^{+} .
$$

The above result is of Abelian nature. Let us mention that to conclude (3.2), it is enough to assume a weaker hypothesis. Indeed, if we only assume the quasiasymptotic behavior of a tempered distribution in the space $\mathcal{S}_{0}^{\prime}(\mathbb{R})$, we are still able to deduce (3.2). Actually, the angular asymptotic behavior over cones with vertex at $x_{0}$ can also be obtained.

Proposition 1. Let $f \in \mathcal{S}^{\prime}(\mathbb{R})$ have quasiasymptotic behavior in $\mathcal{S}_{0}^{\prime}(\mathbb{R})$,

$$
f\left(x_{0}+\varepsilon x\right) \sim \varepsilon^{\alpha} L(\varepsilon) g(x) \quad \text { as } \varepsilon \rightarrow 0^{+} \text {in } \mathcal{S}_{0}^{\prime}(\mathbb{R}) .
$$

Then, given any $0<\sigma \leq \pi / 2$ and $r>0$, we have

$$
\mathcal{W}_{\psi} f\left(x_{0}+\varepsilon r \cos \vartheta, \varepsilon r \sin \vartheta\right) \sim \varepsilon^{\alpha} L(\varepsilon) \mathcal{W}_{\psi} g(r \cos \vartheta, r \sin \vartheta), \quad \varepsilon \rightarrow 0^{+}
$$

uniformly for $\sigma \leq \vartheta \leq \pi-\sigma$.

Proof. In view of (3.3), the Banach-Steinhaus theorem and the compactness of the set

$$
\mathfrak{C}_{\sigma}=\left\{\frac{1}{\sin \vartheta} \bar{\psi}\left(\frac{\cdot-\cos \vartheta}{\sin \vartheta}\right) \in \mathcal{S}_{0}(\mathbb{R}): \sigma \leq \vartheta \leq \pi-\sigma\right\}
$$


we have, as $\varepsilon \rightarrow 0^{+}$,

$$
\begin{aligned}
\mathcal{W}_{\psi} f\left(x_{0}+\varepsilon r \cos \vartheta, \varepsilon r \sin \vartheta\right) & =\left\langle f\left(x_{0}+\varepsilon r \cos \vartheta+\varepsilon r \sin \vartheta x\right), \bar{\psi}(x)\right\rangle \\
& =\left\langle f\left(x_{0}+\varepsilon r x\right), \frac{1}{\sin \vartheta} \bar{\psi}\left(\frac{x-\cos \vartheta}{\sin \vartheta}\right)\right\rangle \\
& \sim(r \varepsilon)^{\alpha} L(r \varepsilon)\left\langle g(x), \frac{1}{\sin \vartheta} \bar{\psi}\left(\frac{x-\cos \vartheta}{\sin \vartheta}\right)\right\rangle \\
& =\varepsilon^{\alpha} L(r \varepsilon)\left\langle g(r x), \frac{1}{\sin \vartheta} \bar{\psi}\left(\frac{x-\cos \vartheta}{\sin \vartheta}\right)\right\rangle \\
& =\varepsilon^{\alpha} L(r \varepsilon) \mathcal{W}_{\psi} g(r \cos \vartheta, r \sin \vartheta) \\
& \sim \varepsilon^{\alpha} L(\varepsilon) \mathcal{W}_{\psi} g(r \cos \vartheta, r \sin \vartheta) .
\end{aligned}
$$

We have a similar assertion at $\infty$ (with a similar proof).

Proposition 2. Let $f \in \mathcal{S}^{\prime}(\mathbb{R})$ have quasiasymptotic behavior at infinity in $\mathcal{S}_{0}^{\prime}(\mathbb{R})$

$$
f(\lambda x) \sim \lambda^{\alpha} L(\lambda) g(x) \quad \text { as } \lambda \rightarrow \infty \text { in } \mathcal{S}_{0}^{\prime}(\mathbb{R}) .
$$

Then, given any $0<\sigma \leq \pi / 2$ and $r>0$, we have

$$
\mathcal{W}_{\psi} f(\lambda r \cos \vartheta, \lambda r \sin \vartheta) \sim \lambda^{\alpha} L(\lambda) \mathcal{W}_{\psi} g(r \cos \vartheta, r \sin \vartheta), \quad \lambda \rightarrow \infty,
$$

uniformly for $\sigma \leq \vartheta \leq \pi-\sigma$.

We now provide some applications of Proposition 1 to distributions given by trigonometric series with exponential gaps. We will obtain (Example 3) a stronger conclusion than the usual nowhere differentiability for the Weierstrass function. For this purpose, we first give Example 2 which is interesting in itself.

Example 2. This example shows how to construct distributions with no point values in the sense of Eojasiewicz (cf. Example 1).

Let $\left\{\lambda_{n}\right\}_{n=0}^{\infty}$ be a lacunary sequence in the sense of Hadamard, that is, a sequence of positive numbers such that there are a $n_{0} \in \mathbb{N}$ and $\sigma>1$ such that $\lambda_{n+1} / \lambda_{n}>\sigma>1, n>n_{0}$. Let $f \in \mathcal{S}^{\prime}(\mathbb{R})$ have a series representation $f(x)=\sum_{n=0}^{\infty} c_{n} e^{i \lambda_{n} x}$, where the series is convergent in $\mathcal{S}^{\prime}(\mathbb{R})$. Furthermore, suppose that at a given $x_{0}$ the point value $f\left(x_{0}\right)$ exists in the sense of Łojasiewicz. Then, by selecting $\psi \in \mathcal{S}_{0}(\mathbb{R})$ with $\operatorname{supp} \overline{\hat{\psi}} \subset\left[\sigma^{-\frac{1}{2}}, \sigma^{\frac{1}{2}}\right]$ and $\overline{\hat{\psi}}(1)=1$, the lacunarity of $\left\{\lambda_{n}\right\}_{n \in \mathbb{N}_{0}}$ implies that for $m$ large enough, $\overline{\hat{\psi}}\left(\lambda_{n} / \lambda_{m}\right)=0$ if $m \neq n$. After a quick calculation, we get

$$
\mathcal{W}_{\psi} f\left(x_{0}, \lambda_{m}^{-1}\right)=\sum_{n=0}^{\infty} c_{n} e^{i \lambda_{n} x_{0}} \overline{\hat{\psi}}\left(\frac{\lambda_{n}}{\lambda_{m}}\right)=c_{m} e^{i \lambda_{m} x_{0}},
$$


So, the existence of the distributional point value $f\left(x_{0}\right)$ and Proposition 1 (with $\alpha=0, L=1, \mathcal{W}_{\psi} 1(0,1)=0$ ) imply that $c_{m} e^{i \lambda_{m} x_{0}}=o(1)$, or,

$$
\lim _{m \rightarrow \infty} c_{m}=0
$$

Therefore, (3.8) is a necessary condition for the existence of the distributional point value of $f$ at $x_{0}$. On the other hand, we have just shown: If (3.8) is violated, then $f$ cannot have distributional point values anywhere. Note that exactly the same argument applies to distributions of the form $\sum_{n=0}^{\infty} c_{n} \cos \left(\lambda_{n} x\right)$ and $\sum_{n=0}^{\infty} c_{n} \sin \left(\lambda_{n} x\right)$. We refer to [44] for related results to this example.

Example 3. A stronger conclusion than the usual nowhere differentiability for Weierstrass's function.

Recall the Weierstrass functions is

$$
w(x)=\sum_{n=0}^{\infty} \gamma^{-n} \cos \left(\beta^{n} x\right), \quad \beta \geq \gamma>1 .
$$

It is continuous and bounded. We look at its first derivative (understood in the distributional sense, of course!)

$$
w^{\prime}(x)=-\sum_{n=0}^{\infty}\left(\frac{\beta}{\gamma}\right)^{n} \sin \left(\beta^{n} x\right) .
$$

Since obviously $(\beta / \gamma)^{n} \neq o(1)$, it follows from Example 2 that $w^{\prime}\left(x_{0}\right)$ does not exist in the sense of Łojasiewicz at any $x_{0} \in \mathbb{R}$. In particular, $w$ is nowhere differentiable.

It should be emphasized that the non-existence of the Eojasiewicz point value of the derivative is much stronger than non-differentiability (example: $h(x)=x^{-1} \sin x$ is not differentiable at 0 , in the ordinary sense, but $h^{\prime}(0)=$ 0 , distributionally). In fact, for the Weierstrass function it means $[26,46]$ that $w$ is nowhere differentiable even in an average sense, namely, for any $x_{0}$ and $m \in \mathbb{N}$

$$
\lim _{x \rightarrow 0} \frac{1}{x^{2}} \int_{0}^{x}\left(w\left(x_{0}+t\right)-w\left(x_{0}\right)\right)\left(1-\frac{t}{x}\right)^{m} \mathrm{~d} t \quad \text { does not exist. }
$$

Example 4. Quasiaymptotics and series with gaps. Let again $f(x)=$ $\sum_{n=0}^{\infty} c_{n} e^{i \lambda_{n} x}$ in $\mathcal{S}^{\prime}(\mathbb{R})$, where $\left\{\lambda_{n}\right\}_{n=0}^{\infty}$ is lacunary. Suppose that

$$
f\left(x_{0}+\varepsilon x\right) \sim \varepsilon^{\alpha} L(\varepsilon) g(x) \text { as } \varepsilon \rightarrow 0^{+} \text {in } \mathcal{S}^{\prime}(\mathbb{R}) .
$$

We will show in this example that if $\alpha \notin \mathbb{N}$, then $g=0$. Since $\operatorname{supp} \hat{f} \subset$ $[0, \infty)$, there must be $C_{+}$such that $\hat{g}(x)=C_{+} x_{+}^{-\alpha-1}$. If we use the same 
wavelet from Example 2 and apply Proposition 1, we conclude that

$$
\begin{aligned}
\lim _{m \rightarrow \infty} \frac{\lambda_{m}^{\alpha} c_{m} e^{i \lambda_{m} x_{0}}}{L\left(1 / \lambda_{m}\right)} & =\lim _{m \rightarrow \infty} \frac{\lambda_{m}^{\alpha} \mathcal{W}_{\psi} f\left(x_{0}, \lambda_{m}^{-1}\right)}{L\left(1 / \lambda_{m}\right)}=\mathcal{W}_{\psi} g(0,1) \\
& =\frac{C_{+}}{2 \pi} \int_{0}^{\infty} \frac{\overline{\hat{\psi}}(x)}{x^{\alpha+1}} \mathrm{~d} x
\end{aligned}
$$

However, we have some degree of freedom over the wavelet, and different choices of $\psi$ lead to different values of the right hand side of the above equation; therefore, $C_{+}=0$. In conclusion, we have shown that the only behavior $f$ can have is $f\left(x_{0}+\varepsilon x\right)=o\left(\varepsilon^{\alpha} L(\varepsilon)\right)$ in $\mathcal{S}^{\prime}(\mathbb{R})$ and

$$
c_{m}=o\left(\frac{L\left(1 / \lambda_{m}\right)}{\lambda_{m}^{\alpha}}\right) \quad \text { as } m \rightarrow \infty
$$

is a necessary condition for this to hold.

3.2. Tauberian Type Results. Our next goal is to provide inverse results for the results of Abelian type given in Propositions 1 and 2, under some natural additional Tauberian conditions. Actually, we characterize below quasiasymptotics in $\mathcal{S}_{0}^{\prime}(\mathbb{R})$ in terms of the wavelet transform. Later, we will use this characterization to study the quasiasymptotic behavior in the space $\mathcal{S}^{\prime}(\mathbb{R})$ (Sections 5 and 6 ). We begin with the case at $\infty$.

Proposition 3. Let $f \in \mathcal{S}_{0}^{\prime}(\mathbb{R})$. Let $\psi \in \mathcal{S}_{0}(\mathbb{R})$ be a wavelet admitting a reconstruction wavelet. The following two conditions:

$$
\lim _{\lambda \rightarrow \infty} \frac{1}{\lambda^{\alpha} L(\lambda)} \mathcal{W}_{\psi} f(\lambda b, \lambda a)=M_{b, a}<\infty,(b, a) \in \mathbb{H},
$$

and the existence of constants $\gamma, \beta, M>0$ such that

$$
\frac{\left|\mathcal{W}_{\psi} f(\lambda b, \lambda a)\right|}{\lambda^{\alpha} L(\lambda)}<M\left(a+\frac{1}{a}\right)^{\gamma}(1+|b|)^{\beta}, \quad(b, a) \in \mathbb{H}, \lambda>1,
$$

are necessary and sufficient for the existence of a distribution $g$ such that

$$
f(\lambda x) \sim \lambda^{\alpha} L(\lambda) g(x) \quad \text { as } \lambda \rightarrow \infty \text { in } \mathcal{S}_{0}^{\prime}(\mathbb{R}) .
$$

In this case we have $M_{b, a}=\mathcal{W}_{\psi} g(b, a),(b, a) \in \mathbb{H}$.

Proof. That (3.10) is necessary follows from the Abelian result, Proposition 2. The necessity of (3.11) follows from the characterization of bounded sets in $\mathcal{S}_{0}^{\prime}(\mathbb{R})$ (c.f. [18, Thm. 28.0.1]). We now focus in showing the converse. Let $\mathfrak{B}=\left\{\psi_{b, a}:=a^{-1} \bar{\psi}\left(a^{-1}(\cdot-b)\right),(b, a) \in \mathbb{H}\right\}$. We claim that the linear span of $\mathfrak{B}$ is dense in $\mathcal{S}_{0}(\mathbb{R})$. Let $h \in \mathcal{S}_{0}^{\prime}(\mathbb{R})$. If we suppose that

$$
\left\langle h(x), \frac{1}{a} \bar{\psi}\left(\frac{x-b}{a}\right)\right\rangle=\mathcal{W}_{\psi} h(b, a)=0, \quad \text { for all }(b, a) \in \mathbb{H},
$$

then, by wavelet desingularization, we have that for every $\phi \in \mathcal{S}_{0}(\mathbb{R})$,

$$
\langle h(x), \phi(x)\rangle=\frac{1}{c_{\psi, \eta}}\left\langle\mathcal{W}_{\psi} h(b, a), \mathcal{W}_{\bar{\eta}} \phi(b, a)\right\rangle=0 ;
$$


and hence $h=0$. Thus, by the Hahn-Banach theorem, we conclude that the linear span of $\mathfrak{B}$ is dense in $\mathcal{S}_{0}(\mathbb{R})$. Furthermore, let $\mathfrak{F}=\left\{f_{\lambda} ; \lambda \geq 1\right\}$ where $f_{\lambda}=f(\lambda \cdot) /\left(\lambda^{\alpha} L(\lambda)\right)$. The estimate (3.11) and the characterization of bounded sets in $\mathcal{S}_{0}^{\prime}(\mathbb{R})$ (c.f. [18, Thm. 28.0.1]) imply that $\mathfrak{F}$ is a bounded family in $\mathcal{S}_{0}^{\prime}(\mathbb{R})$, which in turn implies, by the Banach-Steinhaus theorem, that $\mathfrak{F}$ is an equicontinuous set. It is known that for equicontinuous sets the pointwise convergence over a complete test space and over some dense subset coincide. But observe that (3.10) exactly gives us the convergence over the linear span of $\mathfrak{B}$; so, for some $g \in \mathcal{S}_{0}^{\prime}(\mathbb{R})$, we have $f_{\lambda} \rightarrow g, \lambda \rightarrow \infty$, in the weak sense.

We now consider the asymptotic behavior at finite points. The Tauberian condition which we shall use may be referred as a Vladimirov-DrozhzhinovZavialov type Tauberian condition. Actually, they have made extensive use of these types of conditions in the study of Tauberian theorems for local behavior of generalized functions in terms of several integral transforms, see $[7,8,47,48]$.

We will use the following lemma in the proof of Proposition 4 below. It essentially allows one to pass from a local Tauberian estimate to a global one. Its proof is technically difficult, and we shall postpone it for the Appendix at the end of the article.

Lemma 1. Let $f \in \mathcal{S}_{0}^{\prime}(\mathbb{R})$. Let $\psi \in \mathcal{S}_{0}(\mathbb{R})$ be a wavelet admitting a reconstruction wavelet. Then

$$
\limsup _{\varepsilon \rightarrow 0^{+}} \sup _{a^{2}+b^{2}=1, a>0} \frac{a^{m}}{\varepsilon^{\alpha} L(\varepsilon)}\left|\mathcal{W}_{\psi} f\left(x_{0}+\varepsilon b, \varepsilon a\right)\right|<\infty,
$$

for some $m \in \mathbb{R}$, if and only if there exist $\gamma, \beta, M>0$ such that

$$
\frac{\left|\mathcal{W}_{\psi} f\left(x_{0}+\varepsilon b, \varepsilon a\right)\right|}{\varepsilon^{\alpha} L(\varepsilon)}<M\left(a+\frac{1}{a}\right)^{\gamma}(1+|b|)^{\beta}, \quad(b, a) \in \mathbb{H}, \varepsilon<1 .
$$

The Tauberian characterization of quasiasymptotics at finite points in $\mathcal{S}_{0}^{\prime}(\mathbb{R})$ is given by the following proposition.

Proposition 4. Let $f \in \mathcal{S}_{0}^{\prime}(\mathbb{R})$. Let $\psi \in \mathcal{S}_{0}(\mathbb{R})$ be a wavelet admitting a reconstruction wavelet. The following two conditions:

$$
\lim _{\varepsilon \rightarrow 0^{+}} \frac{1}{\varepsilon^{\alpha} L(\varepsilon)} \mathcal{W}_{\psi} f\left(x_{0}+\varepsilon b, \varepsilon a\right)=M_{b, a}<\infty, \quad a^{2}+b^{2}=1, a>0,
$$

exists, and the existence of $m \in \mathbb{N}$ such that the Tauberian estimate (3.13) holds, are necessary and sufficient for the existence of a distribution $g$ such that

$$
f\left(x_{0}+\varepsilon x\right) \sim \varepsilon^{\alpha} L(\varepsilon) g(x) \quad \text { as } \varepsilon \rightarrow 0^{+} \text {in } \mathcal{S}_{0}^{\prime}(\mathbb{R}) .
$$

In this case we have $M_{b, a}=\mathcal{W}_{\psi} g(b, a)$. 
Proof. Let us first show that (3.15) is valid for all $(b, a) \in \mathbb{H}$. Indeed, for $(b, a) \in \mathbb{H}$ fixed, write $b=r \cos \vartheta$ and $a=r \sin \vartheta$, with $r>0$ and $0<\vartheta<\pi$. Then we have that

$$
\begin{aligned}
\lim _{\varepsilon \rightarrow 0^{+}} \frac{\mathcal{W}_{\psi} f(\varepsilon b, \varepsilon a)}{\varepsilon^{\alpha} L(\varepsilon)} & =\lim _{\varepsilon \rightarrow 0^{+}} \frac{\mathcal{W}_{\psi} f(\varepsilon r \cos \vartheta, \varepsilon r \sin \vartheta)}{\varepsilon^{\alpha} L(\varepsilon)} \\
& =r^{\alpha} \lim _{\varepsilon \rightarrow 0^{+}} \frac{L(\varepsilon)}{L(\varepsilon / r)} \frac{\mathcal{W}_{\psi} f(\varepsilon \cos \vartheta, \varepsilon \sin \vartheta)}{\varepsilon^{\alpha} L(\varepsilon)}=r^{\alpha} M_{\cos \vartheta, \sin \vartheta} .
\end{aligned}
$$

So, we are in the right to write $M_{b, a}:=r^{\alpha} M_{\cos \vartheta} \sin \vartheta$. By Lemma 1 , the estimate (3.13) implies the stronger Tauberian estimate (3.14). Observe that having now (3.14) and (3.15) valid for all $(b, a) \in \mathbb{H}$, the arguments given in the proof of Proposition 3 may lead us to the converse, but we choose to present an alternative version of the proof.

Let $\eta$ be a reconstruction wavelet for $\psi$. Notice that the function given by $J(b, a)=M_{b, a},(b, a) \in \mathbb{H}$, is measurable and satisfies the estimate

$$
|J(b, a)|=\left|M_{b, a}\right|<M\left(a+\frac{1}{a}\right)^{\gamma}(1+|b|)^{\beta}, \quad(b, a) \in \mathbb{H},
$$

hence it is in $\mathcal{S}^{\prime}(\mathbb{H})$. Moreover, because of (3.15) and (3.14), we can use Lebesgue dominated convergence theorem and the wavelet desingularization formula to conclude that for each $\phi \in \mathcal{S}_{0}(\mathbb{R})$

$$
\begin{aligned}
\lim _{\varepsilon \rightarrow 0^{+}}\left\langle\frac{f(\varepsilon x)}{\varepsilon^{\alpha} L(\varepsilon)}, \phi(x)\right\rangle & =\frac{1}{c_{\psi, \eta}} \lim _{\varepsilon \rightarrow 0^{+}} \int_{0}^{\infty} \int_{-\infty}^{\infty} \frac{\mathcal{W}_{\psi} f(\varepsilon b, \varepsilon a)}{\varepsilon^{\alpha} L(\varepsilon)} \mathcal{W}_{\bar{\eta}} \phi(b, a) \frac{\mathrm{d} b \mathrm{~d} a}{a} \\
& =\frac{1}{c_{\psi, \eta}} \int_{0}^{\infty} \int_{-\infty}^{\infty} M_{b, a} \mathcal{W}_{\bar{\eta}} \phi(b, a) \frac{\mathrm{d} b \mathrm{~d} a}{a}
\end{aligned}
$$

Since the last limit exists for each $\phi \in \mathcal{S}_{0}(\mathbb{R})$, it follows that $f$ has quasiasymptotic behavior in the space $\mathcal{S}_{0}^{\prime}(\mathbb{R})$ and that $g$ satisfies (3.16) and $M_{b, a}=\mathcal{W}_{\psi} g(b, a)$.

In conclusion, we have characterized the quasiasymptotic behavior of distributions in the space $\mathcal{S}_{0}^{\prime}(\mathbb{R})$ in terms of the asymptotic behavior of the wavelet transform at approaching points of the boundary. Our main aim is now to extend these results to $\mathcal{S}^{\prime}(\mathbb{R})$, that is, we want to give Tauberian theorems for quasiasymptotics (in $\mathcal{S}^{\prime}(\mathbb{R})$ ) at points and infinity of tempered distributions in terms of the behavior of the wavelet transform. We have reduced this question to the following one: If $f \in \mathcal{S}^{\prime}(\mathbb{R})$ has quasiasymptotics at $x=x_{0}$ or $x=\infty$ in $\mathcal{S}_{0}^{\prime}(\mathbb{R})$, what can we say about the existence of the quasiasymptotics of $f$ at $x=x_{0}$ or $x=\infty$ in $\mathcal{S}^{\prime}(\mathbb{R})$ ? A complete answer to this question will be discussed in the next section.

\section{Quasiasymptotic eXtension from $\mathcal{S}_{0}^{\prime}(\mathbb{R})$ to $\mathcal{S}^{\prime}(\mathbb{R})$.}

We reformulate the problem with the aid of the Fourier transform.

Let $\mathcal{S}\left(\mathbb{R}_{+}\right)$, respectively $\mathcal{S}\left(\mathbb{R}_{-}\right)$, be the closed subspace of $\mathcal{S}(\mathbb{R})$ consisting of functions having support in $\overline{\mathbb{R}}_{+}$, respectively $\overline{\mathbb{R}}_{-}$. Note $\mathcal{F}\left(\mathcal{S}_{+}(\mathbb{R})\right)=$ 
$\mathcal{S}\left(\mathbb{R}_{+}\right), \mathcal{F}\left(\mathcal{S}_{-}(\mathbb{R})\right)=\mathcal{S}\left(\mathbb{R}_{-}\right)$. The space $\mathcal{D}\left(\mathbb{R}_{+}\right)$has different nature than $\mathcal{S}\left(\mathbb{R}_{+}\right)$; it is defined as the set of those elements of $\phi \in \mathcal{D}(\mathbb{R})$ such that $\operatorname{supp} \phi \subset \mathbb{R}_{+}\left(\right.$not $\left.\overline{\mathbb{R}}_{+}\right)$. Similarly for $\mathcal{D}\left(\mathbb{R}_{-}\right)$. Their dual spaces are then $\mathcal{S}^{\prime}\left(\mathbb{R}_{-}\right)=\left(\mathcal{S}\left(\mathbb{R}_{-}\right)\right)^{\prime}, \mathcal{S}^{\prime}\left(\mathbb{R}_{+}\right)=\left(\mathcal{S}\left(\mathbb{R}_{+}\right)\right)^{\prime}, \mathcal{D}^{\prime}\left(\mathbb{R}_{-}\right)=\left(\mathcal{D}\left(\mathbb{R}_{-}\right)\right)^{\prime}$ and $\mathcal{D}^{\prime}\left(\mathbb{R}_{+}\right)=$ $\left(\mathcal{D}\left(\mathbb{R}_{+}\right)\right)^{\prime}$. Let $\mathbb{R}_{0}=\mathbb{R} \backslash\{0\}$. We also consider the spaces $\mathcal{D}\left(\mathbb{R}_{0}\right), \mathcal{S}\left(\mathbb{R}_{0}\right):=$ $\mathcal{S}\left(\mathbb{R}_{-}\right) \oplus \mathcal{S}\left(\mathbb{R}_{+}\right)$and their dual spaces $\mathcal{D}^{\prime}\left(\mathbb{R}_{0}\right)$ and $\mathcal{S}^{\prime}\left(\mathbb{R}_{0}\right)$, respectively.

The problem of extending distributions from $\mathcal{S}_{0}^{\prime}(\mathbb{R})$ to $\mathcal{S}^{\prime}(\mathbb{R})$, together with its asymptotic properties, can be reduced to that of extending distributions from $\mathcal{S}^{\prime}\left(\mathbb{R}_{0}\right)$ to $\mathcal{S}^{\prime}(\mathbb{R})$. For $\mathcal{S}_{0}^{\prime}(\mathbb{R})$ different extensions to $\mathcal{S}^{\prime}(\mathbb{R})$ differ by polynomials, and on $\mathcal{S}^{\prime}\left(\mathbb{R}_{0}\right)$ extensions to $\mathcal{S}^{\prime}(\mathbb{R})$ differ by distributions concentrate at the origin, i.e., finite sums of $\delta$, the Dirac delta distribution, and its derivatives. Indeed, the images under Fourier transform of $\mathcal{S}_{+}^{\prime}(\mathbb{R})$ and $\mathcal{S}_{-}^{\prime}(\mathbb{R})$ are $\mathcal{F}\left(\mathcal{S}_{+}^{\prime}(\mathbb{R})\right)=\mathcal{S}^{\prime}\left(\mathbb{R}_{+}\right)$and $\mathcal{F}\left(\mathcal{S}_{-}^{\prime}(\mathbb{R})\right)=\mathcal{S}^{\prime}\left(\mathbb{R}_{-}\right)$, respectively; finally the image of $\mathcal{S}_{0}^{\prime}(\mathbb{R})$ under Fourier transform is $\mathcal{S}^{\prime}\left(\mathbb{R}_{0}\right)$.

We first analyze quasiasymptotics at finite points. Suppose $f \in \mathcal{S}^{\prime}(\mathbb{R})$ and

$$
f\left(x_{0}+\varepsilon x\right) \sim \varepsilon^{\alpha} L(\varepsilon) g(x) \quad \text { as } \varepsilon \rightarrow 0^{+} \text {in } \mathcal{S}_{0}^{\prime}(\mathbb{R}),
$$

then if we take Fourier transform and replace $\varepsilon=\lambda^{-1}$, we obtain the equivalent expression

$$
e^{i \lambda x_{0} x} \hat{f}(\lambda x) \sim \lambda^{-1-\alpha} L\left(\lambda^{-1}\right) \hat{g}(x) \quad \text { as } \lambda \rightarrow \infty \text { in } \mathcal{S}^{\prime}\left(\mathbb{R}_{0}\right) .
$$

Therefore the problem we are addressing is equivalent to the problem of determining the quasiasymptotic behavior of a tempered distribution at infinity upon knowledge of quasiasymptotics at infinity in $\mathcal{S}^{\prime}\left(\mathbb{R}_{0}\right)$. Since $\mathcal{S}^{\prime}\left(\mathbb{R}_{0}\right)=\mathcal{S}^{\prime}\left(\mathbb{R}_{-}\right) \oplus \mathcal{S}^{\prime}\left(\mathbb{R}_{+}\right)$is enough to work in the space $\mathcal{S}^{\prime}\left(\mathbb{R}_{+}\right)$. We will consider a slightly more general problem. The problem is basically solved by the results of [42, Thm. 4.1-4.3], we state them and add new information below.

We want to make some comments about extension of distributions initially defined in $\mathbb{R}_{0}$ to $\mathbb{R}$. Observe that this problem is of vital importance for renormalization procedures in quantum field theory $([2,24,49,50])$. It also has much relevance to the study of singular integral equations on spaces of distributions [14]. For simplicity, we discuss the problem of extending a distribution from $\mathbb{R}_{+}$to $\mathbb{R}$.

The spaces $\mathcal{D}\left(\overline{\mathbb{R}}_{+}\right)$and $\mathcal{S}\left(\overline{\mathbb{R}}_{+}\right)$are defined as the restriction of the corresponding test functions to $\overline{\mathbb{R}}_{+}$. Their dual spaces $\mathcal{D}^{\prime}\left(\overline{\mathbb{R}}_{+}\right)$and $\mathcal{S}^{\prime}\left(\overline{\mathbb{R}}_{+}\right)$ are identifiable [47, pp.13-14] with the spaces of distributions and tempered distributions supported by $\overline{\mathbb{R}}_{+}$, respectively. Therefore, in discussing extensions of distributions defined on $\mathbb{R}_{+}$to $\mathbb{R}$ is enough to consider the extension to $\overline{\mathbb{R}}_{+}$. In general, it is not true that a distribution $f_{0} \in \mathcal{D}^{\prime}\left(\mathbb{R}_{+}\right)$should have an extension to $\mathcal{D}^{\prime}\left(\overline{\mathbb{R}}_{+}\right)$. The necessary and sufficient conditions [15] for a distribution $f_{0} \in \mathcal{D}^{\prime}\left(\mathbb{R}_{+}\right)$to admit extensions to $\mathcal{D}^{\prime}\left(\overline{\mathbb{R}}_{+}\right)$is the existence of $\beta \in \mathbb{R}$ such that

$$
f_{0}(\varepsilon x)=O\left(\varepsilon^{\beta}\right) \quad \text { as } \varepsilon \rightarrow 0^{+} \text {in } \mathcal{D}^{\prime}\left(\mathbb{R}_{+}\right) .
$$


We call $f_{0} \in \mathcal{D}^{\prime}\left(\mathbb{R}_{+}\right)$extendable to $\overline{\mathbb{R}}_{+}$if $(4.3)$ holds. In relation to the extendable distributions, the notation $\mathcal{D}_{31}\left(\mathbb{R}_{+}\right)$is used in $[14$, p.179] for those test functions from $\mathcal{D}(\mathbb{R})$ having support on $\overline{\mathbb{R}}_{+}$. Its dual is $\mathcal{D}_{31}^{\prime}\left(\mathbb{R}_{+}\right)$. Notice that $\mathcal{D}\left(\mathbb{R}_{+}\right)$is dense in $\mathcal{D}_{31}\left(\mathbb{R}_{+}\right)$; consequently, $\mathcal{D}_{31}^{\prime}\left(\mathbb{R}_{+}\right) \subset \mathcal{D}^{\prime}\left(\mathbb{R}_{+}\right)$. The space $\mathcal{D}_{31}\left(\mathbb{R}_{+}\right)$is closed in $\mathcal{D}\left(\overline{\mathbb{R}}_{+}\right)$; hence every distribution of $\mathcal{D}_{31}^{\prime}\left(\mathbb{R}_{+}\right)$, in view of Hahn-Banach theorem, admits an extension to $\mathcal{D}^{\prime}\left(\overline{\mathbb{R}}_{+}\right)$. Moreover, $\mathcal{D}_{31}^{\prime}\left(\mathbb{R}_{+}\right)$coincides with the extendable distributions from $\mathcal{D}^{\prime}\left(\mathbb{R}_{+}\right)$.

At extending distributions and asymptotic relations, new terms in the asymptotics could appear. In fact, for some cases, we need to consider the so called associate asymptotically homogeneous functions from [42, 46]. A positive measurable function $c$ is said to be associate asymptotically homogeneous of degree zero at infinity, resp. at the origin, with respect to a slowly varying function $L$ if it is defined in some interval of the form $[A, \infty)$ (resp. $(0, A]), A>0$, and there exists $\beta$ such that

$$
c(a x)=c(x)+\beta L(x) \log a+o(L(x)), \quad x \rightarrow \infty \quad\left(\text { resp. } x \rightarrow 0^{+}\right),
$$

for each $a>0$. These functions are also known as de Haan functions [1].

After all these preliminaries, we can state our first theorem.

Theorem 1. Let $f_{0} \in \mathcal{D}^{\prime}\left(\mathbb{R}_{+}\right)$be an extendable distribution to $\overline{\mathbb{R}}_{+}$. Let $\alpha \in \mathbb{R}$ and $L$ be slowly varying at infinity. Suppose that

$$
f_{0}(\lambda x) \sim \lambda^{\alpha} L(\lambda) g(x) \text { as } \lambda \rightarrow \infty \text { in } \mathcal{D}^{\prime}\left(\mathbb{R}_{+}\right) .
$$

Then $f_{0} \in \mathcal{S}^{\prime}\left(\mathbb{R}_{+}\right)$and the quasiasymptotics holds in $\mathcal{S}^{\prime}\left(\mathbb{R}_{+}\right)$. Moreover, let $f \in \mathcal{S}^{\prime}\left(\overline{\mathbb{R}}_{+}\right)$be any extension of $f_{0}$.

(i) If $\alpha>-1$, then $f$ has the quasiasymptotic behavior (4.5) in $\mathcal{S}^{\prime}(\mathbb{R})$.

(ii) If $\alpha<-1$ and $\alpha \notin \mathbb{Z}_{-}$, then there exist constants $a_{0}, \ldots, a_{n-1}, n<-\alpha$, such that

$$
f(\lambda x)=\sum_{j=0}^{n-1} a_{j} \frac{\delta^{(j)}(x)}{\lambda^{j+1}}+\lambda^{\alpha} L(\lambda) g(x)+o\left(\lambda^{\alpha} L(\lambda)\right)
$$

as $\lambda \rightarrow \infty$ in $\mathcal{S}^{\prime}(\mathbb{R})$. The constants depend on the choice of the extension $f$.

(iii) If $\alpha=-k, k \in \mathbb{Z}_{+}$, then $g$ is of the form $g(x)=C \operatorname{Pf}\left(H(x) / x^{k}\right)$ and there are $(k-1)$ constants $a_{0}, \ldots, a_{k-2}$ and an associate asymptotically homogeneous function $c$ of degree 0 with respect to $L$ satisfying

$$
c(a x)=c(x)+\frac{(-1)^{k-1}}{(k-1) !} C L(x) \log a+o(L(x)), \quad x \rightarrow \infty,
$$

such that

$$
f(\lambda x)=C \frac{L(\lambda)}{\lambda^{k}} \operatorname{Pf}\left(\frac{H(x)}{x^{k}}\right)+\frac{c(\lambda)}{\lambda^{k}} \delta^{(k-1)}(x)+\sum_{j=0}^{k-2} a_{j} \frac{\delta^{(j)}(x)}{\lambda^{j+1}}+o\left(\frac{L(\lambda)}{\lambda^{k}}\right)
$$


as $\lambda \rightarrow \infty$ in $\mathcal{S}^{\prime}(\mathbb{R})$. The constants and the function $c$ depend on the choice of the extension $f$.

Proof. The statements (i), (ii), and (iii), are direct consequences of [42, Thm.4.1-4.3], it also follows that $f$ is a tempered distribution. Since $f_{0}$ is the restriction of $f$ to $\mathcal{D}\left(\mathbb{R}_{+}\right)$, we infer that $f_{0} \in \mathcal{S}^{\prime}\left(\mathbb{R}_{+}\right)$; furthermore, by the same reason, the quasiasymptotic behavior (4.5) holds in that space.

Corollary 1. Let $f \in \mathcal{S}^{\prime}(\mathbb{R})$. Let $\alpha \in \mathbb{R}, x_{0} \in \mathbb{R}$ and $L$ be slowly varying at the origin. Suppose that

$$
f\left(x_{0}+\varepsilon x\right) \sim \varepsilon^{\alpha} L(\varepsilon) g(x) \quad \text { as } \varepsilon \rightarrow 0^{+} \text {in } \mathcal{S}_{0}^{\prime}(\mathbb{R}) .
$$

(i) If $\alpha<0$, then $f$ has the quasiasymptotic behavior (4.9) in $\mathcal{S}^{\prime}(\mathbb{R})$.

(ii) If $\alpha>0$ and $\alpha \notin \mathbb{Z}_{+}$, then there exists a polynomial $p$, of degree less than $\alpha$, such that

$$
f\left(x_{0}+\varepsilon x\right)=p(\varepsilon x)+\varepsilon^{\alpha} L(\varepsilon) g(x)+o\left(\varepsilon^{\alpha} L(\varepsilon)\right) \quad \text { as } \varepsilon \rightarrow 0^{+} \text {in } \mathcal{S}^{\prime}(\mathbb{R}) .
$$

(iii) If $\alpha=k, k \in \mathbb{N}$, then $g$ is of the form $g(x)=C_{-} x_{-}^{k}+C_{+} x_{+}^{k}+\beta x^{k} \log |x|$, and there are a polynomial $p$ of degree at most $(k-1)$ and an associate asymptotically homogeneous function $c$, satisfying (4.4), such that

$$
f\left(x_{0}+\varepsilon x\right)=p(\varepsilon x)+c(\varepsilon) \varepsilon^{k} x^{k}+\varepsilon^{k} L(\varepsilon) g(x)+o\left(\varepsilon^{k} L(\varepsilon)\right) .
$$

as $\varepsilon \rightarrow 0^{+}$in $\mathcal{S}^{\prime}(\mathbb{R})$.

Proof. As in (4.2), take Fourier transform to $f\left(x_{0}+\cdot\right)$. In $\mathcal{S}_{0}^{\prime}(\mathbb{R})$, we have unique decompositions $e^{i x_{0} x} \hat{f}=f_{-}+f_{+}$and $\hat{g}=g_{-}+g_{+}$, where $f_{ \pm}, g_{ \pm} \in \mathcal{S}^{\prime}\left(\mathbb{R}_{ \pm}\right)$. A direct application of Theorem 1 to $f_{ \pm}, g_{ \pm}$and $L(1 / \lambda)$ yields the result on the Fourier side.

We now extend Theorem 1 to quasiasymptotics at the origin. The proof of the next theorem is an adaptation of the arguments from [42, Sec.4] to our context.

Theorem 2. Let $f_{0} \in \mathcal{D}^{\prime}\left(\mathbb{R}_{+}\right)$have the quasiasymptotic behavior

$$
f_{0}(\varepsilon x) \sim \varepsilon^{\alpha} L(\varepsilon) g(x) \quad \text { as } \varepsilon \rightarrow 0^{+} \text {in } \mathcal{D}^{\prime}\left(\mathbb{R}_{+}\right) .
$$

Then $f_{0}$ is extendable to $\overline{\mathbb{R}}_{+}$. Moreover, if $f \in \mathcal{D}^{\prime}\left(\overline{\mathbb{R}}_{+}\right)$is an extension of $f_{0}$ to $\overline{\mathbb{R}}_{+}$, one has that:

(i) If $\alpha \notin \mathbb{Z}_{-}$, then there exist constants $a_{0}, a_{1}, \ldots, a_{m-1}$ such that

$$
f(\varepsilon x)=\varepsilon^{\alpha} L(\varepsilon) g(x)+\sum_{j=0}^{m-1} a_{j} \frac{\delta^{(j)}(x)}{\varepsilon^{j+1}}+o\left(\varepsilon^{\alpha} L(\varepsilon)\right)
$$

as $\varepsilon \rightarrow 0^{+}$in $\mathcal{D}^{\prime}(\mathbb{R})$.

(ii) If $\alpha=-k, k \in \mathbb{Z}_{+}$, then $g$ is of the form $g(x)=C \operatorname{Pf}\left(H(x) / x^{k}\right)$ and there exist an associate asymptotically homogeneous function $c$ satisfying

$$
c(a x)=c(x)+\frac{(-1)^{k-1}}{(k-1) !} C L(x) \log a+o(L(x)), \quad x \rightarrow 0^{+},
$$


for each $a>0$, and constants $a_{k}, a_{k+1}, \ldots, a_{m-1}$ such that

$$
f(\varepsilon x)=C \frac{L(\varepsilon)}{\varepsilon^{k}} \operatorname{Pf}\left(\frac{H(x)}{x^{k}}\right)+\frac{c(\varepsilon)}{\varepsilon^{k}} \delta^{(k-1)}(x)+\sum_{j=k-1}^{m-1} a_{j} \frac{\delta^{(j)}(x)}{\varepsilon^{j+1}}+o\left(\frac{L(\varepsilon)}{\varepsilon^{k}}\right)
$$

as $\varepsilon \rightarrow 0^{+}$in $\mathcal{D}^{\prime}(\mathbb{R})$.

Furthermore, if one assume that $f_{0} \in \mathcal{S}^{\prime}\left(\mathbb{R}_{+}\right)$, then $f \in \mathcal{S}^{\prime}\left(\overline{\mathbb{R}}_{+}\right)$and the asymptotic expansions (4.13) and (4.15) hold in $\mathcal{S}^{\prime}(\mathbb{R})$.

Proof. (i) Since $\alpha$ is not a negative integer and the quasiasymptotic behavior (4.12) holds on the positive part of the real line we have that

$$
g(x)=C \frac{x_{+}^{\alpha}}{\Gamma(\alpha+1)} \text { for some constant } C .
$$

In $\left[46\right.$, Prop.4.1], we may replace the space $\mathcal{D}^{\prime}(\mathbb{R})$ by $\mathcal{D}^{\prime}\left(\mathbb{R}_{+}\right)$; as in the proof of [46, Thm.4.2], we have that there are a positive integer $m>-\alpha$, an $m$ primitive $F_{m}$ of $f_{0}$ in $\mathcal{D}^{\prime}\left(\mathbb{R}_{+}\right)$, which is continuous on the interval $(0,1)$, and a polynomial $p$ such that

$$
F_{m}(\varepsilon x)=C_{+} L(\varepsilon) \frac{(\varepsilon x)_{+}^{\alpha+m}}{\Gamma(\alpha+m+1)}+o\left(\varepsilon^{\alpha+m} L(\varepsilon)\right)
$$

as $\varepsilon \rightarrow 0^{+}$, uniformly for $x \in[1 / 2,1]$. Setting $x=1$ and replacing $x$ by $\varepsilon$, we obtain that

$$
F_{m}(x)=C_{+} L(x) \frac{x_{+}^{\alpha+m}}{\Gamma(\alpha+m+1)}+o\left(x^{\alpha+m} L(x)\right),
$$

in the ordinary sense. Therefore, $F$ is actually continuous on $[0,1)$ and the asymptotic formula (4.16) holds in $\mathcal{D}^{\prime}(\mathbb{R})$. Let $f_{1}=F_{m}^{(m)}$, differentiating (4.16) $m$-times, we see that $f_{1}$ has the quasiasymptotic behavior (4.12) in $\mathcal{D}^{\prime}(\mathbb{R})$, and $f_{1}$ is an extension of $f_{0}$. The rest follows from the observation that $f-f_{1}$ is a distribution concentrated at the origin, and hence it is a sum of the Dirac delta distribution and its derivatives.

(ii) Let us observe that if we take the space $\mathcal{D}^{\prime}\left(\mathbb{R}_{+}\right)$instead of $\mathcal{D}^{\prime}(\mathbb{R})$ in $[46$, Prop.3.1] and [46, Prop.4.3], they still hold. Hence, the arguments given in [46, Section 5] are still applicable to conclude the existence of $m \in \mathbb{N}$, $m>k$, and $F_{m}$, an $m$-primitive of $f_{0}$ in $\mathcal{D}^{\prime}\left(\mathbb{R}_{+}\right)$, which is continuous on the interval $(0,1)$, such that

$F_{m}(x)=c_{1}(x) \frac{x^{m-k}}{(m-k) !}-C L(x) \frac{x^{m-k}}{(m-k) !} \sum_{j=1}^{m-k} \frac{1}{j}+o\left(x^{m-k} L(x)\right), \quad x \rightarrow 0^{+}$,

in the ordinary sense, where the function $c_{1}$ satisfies (4.14). Notice that $F_{m}$ is then continuous in $[0,1)$. By [46, Lemm.5.1], we have

$$
F_{m}(\varepsilon x)=c_{1}(\varepsilon) \frac{(\varepsilon x)_{+}^{m-k}}{(m-k) !}+C \varepsilon^{m-k} L(\varepsilon) l_{m-k}(x) H(x)+o\left(\varepsilon^{m-k} L(\varepsilon)\right),
$$


as $\varepsilon \rightarrow 0^{+}$in $\mathcal{D}^{\prime}(\mathbb{R})$, where

$$
l_{m-k}(x)=\frac{x^{m-k}}{(m-k) !} \log x-\frac{x^{m-k}}{(m-k) !} \sum_{j=1}^{m-k} \frac{1}{j} .
$$

Differentiating the last expression $(m-k)$-times, we get

$$
F_{m}^{(m-k)}(\varepsilon x)=c_{1}(\varepsilon) H(x)+C L(\varepsilon) H(x) \log x+o(L(\varepsilon)),
$$

as $\varepsilon \rightarrow 0^{+}$in $\mathcal{D}^{\prime}(\mathbb{R})$. Set now $f_{1}=F_{m}^{(m)}, k$ more differentiations of $(4.17)$ and the formula

$$
\frac{d^{k-1}}{d x^{k-1}}\left(\operatorname{Pf}\left(\frac{H(x)}{x}\right)\right)=(-1)^{k-1}(k-1) ! \operatorname{Pf}\left(\frac{H(x)}{x^{k}}\right)-\delta^{(k-1)}(x) \sum_{j=1}^{k} \frac{1}{j},
$$

imply that

$$
f_{1}(\varepsilon x)=C \frac{L(\varepsilon)}{\varepsilon^{k}} \operatorname{Pf}\left(\frac{H(x)}{x^{k}}\right)+\frac{c(\varepsilon)}{\varepsilon^{k}} \delta^{(k-1)}(x)+o\left(\frac{L(\varepsilon)}{\varepsilon^{k}}\right)
$$

with $c(x)=c_{1}(x)-C L(x) \sum_{j=1}^{k} \frac{1}{j}$. Since $f_{1}$ is an extension of $f_{0}$, then $f-f_{1}$ is concentrated at the origin, and hence we obtain (4.15).

The same arguments given in the proof of Corollary 1, but now using Theorem 2, lead to the following corollary.

Corollary 2. Let $f \in \mathcal{S}^{\prime}(\mathbb{R})$. Let $L$ be slowly varying at infinity and $\alpha \in \mathbb{R}$. Suppose that

$$
f(\lambda x) \sim \lambda^{\alpha} L(\lambda) g(x) \quad \text { as } \lambda \rightarrow \infty \text { in } \mathcal{S}_{0}^{\prime}(\mathbb{R}) .
$$

(i) If $\alpha \notin \mathbb{N}$, then there exists a polynomial $p$, which may be chosen to be divisible by $x^{\max \{0,[\alpha]+1\}}$, such that

$$
f(\lambda x)=p(\lambda x)+\lambda^{\alpha} L(\lambda) g(x)+o\left(\lambda^{\alpha} L(\lambda)\right) \quad \text { as } \lambda \rightarrow \infty \text { in } \mathcal{S}^{\prime}(\mathbb{R}) .
$$

(ii) If $\alpha=k, k \in \mathbb{N}$, then $g$ is of the form $g(x)=C_{-} x_{-}^{k}+C_{+} x_{+}^{k}+\beta x^{k} \log |x|$, and there are a polynomial $p$, which may be chosen divisible by $x^{k+1}$, and an associate asymptotically homogeneous function c, satisfying (4.4), such that

$$
f\left(x_{0}+\lambda x\right)=p(\lambda x)+c(\lambda) \lambda^{k} x^{k}+\lambda^{k} L(\lambda) g(x)+o\left(\lambda^{k} L(\lambda)\right),
$$

as $\lambda \rightarrow \infty$ in $\mathcal{S}^{\prime}(\mathbb{R})$.

\section{TAUBERIAN THEOREMS FOR QUASIASYMPTOTICS AT POINTS.}

As a consequence of our analysis from Sections 3 and 4, we obtain the Tauberian theorems for quasiasymptotics at points of tempered distributions. The proofs of the next three theorems follow at once by applying Proposition 4 and Corollary 1. 
Theorem 3. Let $f \in \mathcal{S}^{\prime}(\mathbb{R})$ and $\alpha<0$. Suppose that the wavelet $\psi \in$ $\mathcal{S}_{0}(\mathbb{R})$ admits a reconstruction wavelet. Necessary and sufficient conditions in terms of the wavelet transform for $f$ to have quasiasymptotic behavior at $x=x_{0}$ of degree $\alpha$ with respect to a slowly varying function $L$ are the existence of the limits

$$
\lim _{\varepsilon \rightarrow 0^{+}} \frac{1}{\varepsilon^{\alpha} L(\varepsilon)} \mathcal{W}_{\psi} f\left(x_{0}+\varepsilon b, \varepsilon a\right)=M_{b, a}<\infty, \quad a^{2}+b^{2}=1, a>0,
$$

and the existence of $m$ such that

$$
\limsup _{\varepsilon \rightarrow 0^{+}} \sup _{a^{2}+b^{2}=1, a>0} \frac{a^{m}}{\varepsilon^{\alpha} L(\varepsilon)}\left|\mathcal{W}_{\psi} f\left(x_{0}+\varepsilon b, \varepsilon a\right)\right|<\infty .
$$

In such a case there is a homogeneous distribution $g$ of degree $\alpha$ such that $M_{b, a}=\mathcal{W}_{\psi} g(b, a)$.

Theorem 4. Let $f \in \mathcal{S}^{\prime}(\mathbb{R})$ and $\alpha>0, \alpha \notin \mathbb{N}$. Suppose that the wavelet $\psi \in \mathcal{S}_{0}(\mathbb{R})$ admits a reconstruction wavelet. Conditions (5.1) and (5.2) are necessary and sufficient for the existence of a polynomial $p$ of degree less than $\alpha$ such that $f-p$ has quasiasymptotic behavior of degree $\alpha$ with respect to $L$ at the point $x=x_{0}$. In such a case there is a homogeneous distribution $g$ of degree $\alpha$ such that $M_{b, a}=\mathcal{W}_{\psi} g(b, a)$.

Theorem 5. Let $f \in \mathcal{S}^{\prime}(\mathbb{R})$ and $k \in \mathbb{N}$. Suppose that the wavelet $\psi \in \mathcal{S}_{0}(\mathbb{R})$ admits a reconstruction wavelet. Conditions (5.1) and (5.2) with $\alpha=k$ are necessary and sufficient for the existence of a distribution of the form $g(x)=C_{-} x_{-}^{k}+C_{+} x_{+}^{k}+\beta x^{k} \log |x|$, a polynomial $p$ of degree at most $(k-1)$, and an associate asymptotically homogeneous function c, satisfying (4.4), such that

$$
f\left(x_{0}+\varepsilon x\right)=p(\varepsilon x)+c(\varepsilon) \varepsilon^{k} x^{k}+\varepsilon^{k} L(\varepsilon) g(x)+o\left(\varepsilon^{k} L(\varepsilon)\right),
$$

as $\varepsilon \rightarrow 0^{+}$in $\mathcal{S}^{\prime}(\mathbb{R})$. Moreover, $M_{b, a}=\mathcal{W}_{\psi} g(b, a)$.

Remark 1. Theorems 3-5 have an interesting variant involving uniformity. Let $\left\{f_{t}\right\}_{t}$ be a family of distributions indexed by $t \in \Lambda$. For instance, in the case of Theorem $4,(5.1)$ and (5.2) with $f=f_{t}$ with uniformity in $t$ are necessary and sufficient for the existence of polynomials $\left\{p_{t}\right\}_{t}$ such that $f_{t}-p_{t}$ have uniform quasiasymptotics with respect to $t$, namely, for each $\varphi \in \mathcal{S}(\mathbb{R})$ there holds

$$
\lim _{\epsilon \rightarrow 0^{+}}\left\langle\frac{f_{t}\left(x_{0}+\varepsilon x\right)-p_{t}(\varepsilon x)}{\varepsilon^{\alpha} L(\varepsilon)}, \varphi(x)\right\rangle=C_{t, \varphi}, \quad \text { uniformly for } t \in \Lambda .
$$

In fact, straightforward modifications of our arguments lead to the desired uniformity. Similar considerations are valid for Theorems 3 and 5. Such results can be used to study local properties of distributions at different points simultaneously, they will be the subject of our further investigations of vector-valued wavelet transforms with applications within various function spaces. 
5.1. Applications of the Tauberian Theorems. We now give an application of our Tauberian theorems. We provide a new proof of Littlewood's Tauberian theorem for the converse of Abel theorem on power series [25].

The celebrated Littlewood's Tauberian theorem states that if

$$
\lim _{y \rightarrow 0^{+}} \sum_{n=0}^{\infty} c_{n} e^{-y n}=\gamma
$$

and if the Tauberian hypothesis $c_{n}=O(1 / n)$ is satisfied, then

$$
\sum_{n=0}^{\infty} c_{n}=\gamma \text {. }
$$

We will give a "wavelet proof" of this theorem based on Theorem 5.

Example 5. Proof. Let $M>0$ be such that $\left|c_{n}\right|<M / n, n \geq 1$. Set

$$
f(x)=\sum_{n=0}^{\infty} c_{n} e^{i n x} \text { and } F(b, a)=F(b+i a)=\sum_{n=0}^{\infty} c_{n} e^{i n(b+i a)} .
$$

Then, $F$ is analytic in $z=b+i a$, for $a>0$. Observe that (5.4) tells us that $F(i a) \rightarrow \gamma$, as $a \rightarrow 0^{+}$. We actually have a stronger assertion under the Tauberian hypothesis, namely, non-tangential convergence.

Lemma 2. There is $C>0$ such that

$$
|F(\varepsilon b, \varepsilon a)|<\frac{C}{a}(1+a)(1+|b|),(b, a) \in \mathbb{H} .
$$

Furthermore, for each $(b, a) \in \mathbb{H}$ we have that

$$
\lim _{\varepsilon \rightarrow 0^{+}} F(\varepsilon b, \varepsilon a)=\gamma .
$$

Proof. Let $s(x)=\sum_{0 \leq n<x} c_{n}$. First, we prove that $s(x)=O(1)$ as $x \rightarrow \infty$. Indeed, by (5.4) and the condition $\left|c_{n}\right| \leq M / n$, we have that for suitable positive constants $C_{0}$ and $C_{1}$

$$
\begin{aligned}
|s(x)| & \leq\left|s(x)-F\left(\frac{i}{x}\right)\right|+C_{0} \leq M \sum_{0<n<x} \frac{1-e^{-\frac{n}{x}}}{n}+M \sum_{x \leq n} \frac{1}{n} e^{-\frac{n}{x}}+C_{0} \\
& \leq \frac{M}{x} \sum_{n<x} 1+\frac{C_{1}}{x} \int_{x}^{\infty} e^{-\frac{t}{x}} \mathrm{~d} t+C_{0}<C, \quad \text { for some } C>0 .
\end{aligned}
$$

We now show (5.6). We have

$$
\begin{gathered}
|F(\varepsilon b, \varepsilon a)|=\left|\int_{0}^{\infty} e^{-\varepsilon t(a-i b)} \mathrm{d} s(t)\right| \\
\leq \varepsilon|a-i b| C \int_{0}^{\infty} e^{-\varepsilon t a} \mathrm{~d} t \leq \frac{C}{a}(1+a)(1+|b|),
\end{gathered}
$$

where $C$ does not depend on $a, b$, and $\varepsilon$. In particular, $F$ is bounded over cones with vertex at the origin. It is well known that for bounded analytic functions the existence of radial limits is equivalent to non-tangential limits [5]; hence (5.7) follows. 
Our plan is to apply Theorem 5 to show the existence of $c$ such that

$$
f(\varepsilon x)=c(\varepsilon)+o(1) \quad \text { in } \mathcal{S}^{\prime}(\mathbb{R}),
$$

and later to use (5.4) and deduce that indeed $f(0)=\gamma$ in the sense of Łojasiewicz (cf. Example 1). This will imply (5.5), as we shall see.

Let $\hat{\psi}_{1}(x)=e^{-|x|-\frac{1}{|x|}}$ and choose $\varphi \in \mathcal{S}(\mathbb{R})$ such that $\hat{\varphi}$ is real-valued and $\hat{\varphi}(x)=e^{-x}$, for $x \geq 0$. Consider the wavelet $\psi=\varphi * \psi_{1}$. If $0<a \leq 1$ and $\varepsilon \leq 1$, we have with a suitable constant $C_{2}$

$$
\left|\mathcal{W}_{\psi} f(\varepsilon b, \varepsilon a)\right| \leq M \sum_{n=1}^{\infty} \frac{1}{n} \hat{\psi}(\varepsilon a n) \leq C_{2} \int_{0}^{\infty} \frac{\hat{\psi}(x)}{x}
$$

so the Tauberian estimate (cf. (5.2)) for the wavelet transform of $f$ has been established. Next, a quick calculation shows that for fixed $(b, a) \in \mathbb{H}$ (here $\left.\varphi_{a}=a^{-1} \check{\varphi}(\cdot / a)\right)$

$$
\mathcal{W}_{\psi} f(b, a)=\int_{-\infty}^{\infty} \bar{\psi}_{1}(t)\left(f * \varphi_{a}\right)(b+a t) \mathrm{d} t=\int_{-\infty}^{\infty} F(b+a t, a) \bar{\psi}_{1}(t) \mathrm{d} t .
$$

Therefore, Lemma 2 gives us the right to apply Lebesgue's dominated convergence theorem to conclude that

$$
\lim _{\varepsilon \rightarrow 0^{+}} \mathcal{W}_{\psi} f(\varepsilon b, \varepsilon a)=\lim _{\varepsilon \rightarrow 0^{+}} \int_{-\infty}^{\infty} F(\varepsilon b+\varepsilon a t, \varepsilon a) \bar{\psi}_{1}(t) \mathrm{d} t=\gamma \int_{-\infty}^{\infty} \bar{\psi}_{1}(t) \mathrm{d} t=0
$$

So, (5.8) follows now from Theorem 5. On the other hand, if we evaluate (5.8) at $\varphi$, we have

$$
\lim _{\varepsilon \rightarrow 0^{+}} c(\varepsilon)=\lim _{\varepsilon \rightarrow 0^{+}}\langle f(\varepsilon x), \varphi(x)\rangle=\lim _{\varepsilon \rightarrow 0^{+}} \sum_{n=0}^{\infty} c_{n} e^{-\varepsilon n}=\gamma .
$$

Our conclusion from this wavelet analysis is that $f(0)=\gamma$, distributionally. Here comes the final step in our argument. Let $\sigma>0$ be an arbitrary positive number. Choose an even test function $\theta \in \mathcal{S}(\mathbb{R})$ such that $0 \leq \hat{\theta} \leq 1$, supp $\hat{\theta} \subset[-1-\sigma, 1+\sigma]$ and $\hat{\theta}(x)=1$, for $x \in[0,1]$. Then, since

$$
\gamma=\lim _{\varepsilon \rightarrow 0^{+}}\langle f(\varepsilon x), \theta(x)\rangle=\lim _{\varepsilon \rightarrow 0^{+}} \sum_{n=0}^{\infty} c_{n} \hat{\theta}(\varepsilon n),
$$

we obtain then

$\limsup _{\varepsilon \rightarrow 0^{+}}\left|\sum_{n \leq \frac{1}{\varepsilon}} c_{n}-\gamma\right| \leq M \limsup _{\varepsilon \rightarrow 0^{+}} \sum_{1<\varepsilon n<1+\sigma} \frac{\hat{\theta}(\varepsilon n)}{n}=M \int_{1}^{1+\sigma} \frac{\hat{\theta}(x)}{x} \mathrm{~d} x<\sigma M$.

Since $\sigma$ was arbitrary, we conclude that $\sum_{n=0}^{\infty} c_{n}=\gamma$, as required.

Remark 2. The presented Tauberian results and Remark 1 give a new approach for the investigations of local properties of functions, for example in the Hölder and Zygmund classes involving 2-microlocal characterizations, 
through their wavelet transforms. We will give examples which correspond to the classical ones in our forthcoming papers.

5.2. Tauberian Conditions for $\alpha \geq 0$. We may formulate Tauberian conditions in order to guarantee quasiasymptotic behavior in the case $\alpha \geq 0$. We also point out that test functions can always be found satisfying the hypothesis of the next two corollaries [11].

Corollary 3. Let the hypotheses of Theorem 4 be satisfied. Let $n=[\alpha]$. Let $\varphi \in \mathcal{S}(\mathbb{R})$ be such that its moments $\mu_{j}:=\int_{-\infty}^{\infty} x^{j} \varphi(x) \mathrm{d} x \neq 0$, for $0 \leq j \leq n$. The condition

$$
\left\langle f\left(x_{0}+\varepsilon x\right), \varphi(x)\right\rangle=O\left(\varepsilon^{\alpha} L(\varepsilon)\right), \quad \varepsilon \rightarrow 0^{+},
$$

implies that $f$ has quasiasymptotic behavior of degree $\alpha$ with respect to $L$ at the point $x=x_{0}$.

Proof. By Theorem 4, there exist $(n+1)$ constants $c_{0}, c_{1}, \ldots, c_{n}$ and a homogeneous distribution $g$ of degree $\alpha$ such that

$$
f(\varepsilon x)=\sum_{j=0}^{n} c_{j} \varepsilon^{j} x^{j}+\varepsilon^{\alpha} L(\varepsilon) g(x)+o\left(\varepsilon^{\alpha} L(\varepsilon)\right) \quad \text { as } \varepsilon \rightarrow 0^{+} \text {in } \mathcal{S}^{\prime}(\mathbb{R}) .
$$

Evaluating the last asymptotic expansion at $\varphi$ and comparing with (5.9), one has that

$$
\sum_{j=0}^{n} \varepsilon^{j} c_{j} \mu_{j}=O\left(\varepsilon^{\alpha} L(\varepsilon)\right)
$$

which readily implies that $c_{j}=0$, for each $0 \leq j \leq n$.

Corollary 4. Let the hypothesis of Theorem 5 be satisfied and $\alpha=k$. Let $\varphi \in \mathcal{S}(\mathbb{R})$ be such that its moments $\mu_{j}:=\int_{-\infty}^{\infty} x^{j} \varphi(x) \mathrm{d} x \neq 0$, for $0 \leq j \leq k$. The condition

$$
\left\langle f\left(x_{0}+\varepsilon x\right), \varphi(x)\right\rangle \sim C \varepsilon^{k} L(\varepsilon), \quad \varepsilon \rightarrow 0^{+},
$$

for some constant $C$, implies that $f$ has quasiasymptotic behavior of degree $k$ with respect to $L$ at the point $x=x_{0}$.

Proof. Comparison between (5.10) and (5.3), evaluated at $\varphi$, gives that the polynomial vanishes and the asymptotic relation

$$
\begin{gathered}
c(\varepsilon) \sim \frac{L(\varepsilon)}{\mu_{k}}\left(C-C_{-} \int_{0}^{\infty} x^{k} \varphi(-x) \mathrm{d} x-C_{+} \int_{0}^{\infty} x^{k} \varphi(x) \mathrm{d} x\right. \\
-\beta \int_{-\infty}^{\infty} x^{k} \varphi(x) \log |x| \mathrm{d} x,
\end{gathered}
$$

from where we obtain the result.

Our next Tauberian theorems makes use of quasiasymptotic boundedness [43] as the Tauberian condition. We call the distribution $f$ quasiasymptotic bounded of degree $\alpha$ at $x=x_{0}$ with respect to a function $L$, slowly varying at the origin, if $f\left(x_{0}+\varepsilon \cdot\right) /\left(\varepsilon^{\alpha} L(\varepsilon)\right)$ is a weak bounded set in $\mathcal{S}^{\prime}(\mathbb{R})$, for $\varepsilon$ 
small enough. In this case we write $f\left(x_{0}+\varepsilon x\right)=O\left(\varepsilon^{\alpha} L(\varepsilon)\right)$ as $\varepsilon \rightarrow 0^{+}$in $\mathcal{S}^{\prime}(\mathbb{R})$.

Theorem 6. Let $f \in \mathcal{S}^{\prime}(\mathbb{R}), x_{0} \in \mathbb{R}, \alpha \notin \mathbb{N}$, and $L$ be a slowly varying function at the origin. Let $\psi \in \mathcal{S}_{0}(\mathbb{R})$ be a wavelet admitting a reconstruction wavelet. Suppose that the following limits exist:

$$
\lim _{\varepsilon \rightarrow 0^{+}} \frac{1}{\varepsilon^{\alpha} L(\varepsilon)} \mathcal{W}_{\psi} f\left(x_{0}+\varepsilon b, \varepsilon a\right)=M_{b, a}<\infty, \quad a^{2}+b^{2}=1, a>0 .
$$

Then, the Tauberian condition

$$
f\left(x_{0}+\varepsilon x\right)=O\left(\varepsilon^{\alpha} L(\varepsilon)\right) \quad \text { as } \varepsilon \rightarrow 0^{+} \text {in } \mathcal{S}^{\prime}(\mathbb{R}),
$$

implies the existence of a homogeneous distribution $g$ of degree $\alpha$ such that $M_{b, a}=\mathcal{W}_{\psi} g(b, a)$ and

$$
f\left(x_{0}+\varepsilon x\right) \sim \varepsilon^{\alpha} L(\varepsilon) g(x) \quad \text { as } \varepsilon \rightarrow 0^{+} \text {in } \mathcal{S}^{\prime}(\mathbb{R}) .
$$

Conversely, the quasiasymptotic behavior (5.13) implies (5.11) and (5.12).

Proof. The converse is clear; indeed, Proposition 1, implies (5.11), and, obviously, quasiasymptotic behavior implies quasiasymptotic boundedness. On the other hand, relation (5.12) holds in particular in $\mathcal{S}_{0}^{\prime}(\mathbb{R})$, hence, the characterization of bounded sets of $\mathcal{S}_{0}^{\prime}(\mathbb{R})[18$, Thm.28.0.1] implies that (5.2) is satisfied. If $\alpha<0$, then Theorem 3 implies (5.13). Now, if $\alpha>0$, we can always select a test function $\varphi$ such that its moments $\mu_{j}:=\int_{-\infty}^{\infty} x^{j} \varphi(x) \mathrm{d} x \neq$ 0 , for $0 \leq j \leq[\alpha]$. But if we evaluate (5.12) at $\varphi$, we obtain (5.9), and thus, Corollary 3 yields the result in this case.

Theorem 7. Let $f \in \mathcal{S}^{\prime}(\mathbb{R}), x_{0} \in \mathbb{R}, k \in \mathbb{N}$, and $L$ be a slowly varying function at the origin. Let $\psi \in \mathcal{S}_{0}(\mathbb{R})$ be a wavelet admitting a reconstruction wavelet. Suppose that the following limits exist:

$$
\lim _{\varepsilon \rightarrow 0^{+}} \frac{1}{\varepsilon^{k} L(\varepsilon)} \mathcal{W}_{\psi} f\left(x_{0}+\varepsilon b, \varepsilon a\right)=M_{b, a}<\infty, \quad a^{2}+b^{2}=1, a>0 .
$$

Then, the Tauberian condition

$$
f\left(x_{0}+\varepsilon x\right)=O\left(\varepsilon^{k} L(\varepsilon)\right) \quad \text { as } \varepsilon \rightarrow 0^{+} \text {in } \mathcal{S}^{\prime}(\mathbb{R}),
$$

implies the existence of a distribution, having the form $g(x)=C_{-} x_{-}^{k}+$ $C_{+} x_{+}^{k}+\beta x^{k} \log |x|$, and an associate asymptotically homogeneous function $c$, satisfying (4.4), such that $M_{b, a}=\mathcal{W}_{\psi} g(b, a)$ and

$$
f\left(x_{0}+\varepsilon x\right)=c(\varepsilon) \varepsilon^{k} x^{k}+\varepsilon^{k} L(\varepsilon) g(x)+o\left(\varepsilon^{k} L(\varepsilon)\right) \quad \text { as } \varepsilon \rightarrow 0^{+} \text {in } \mathcal{S}^{\prime}(\mathbb{R}) .
$$

Moreover, $c(\varepsilon)=O(L(\varepsilon))$. Additionally, if there exists $\varphi \in \mathcal{S}^{\prime}(\mathbb{R})$ satisfying (5.10) and having non-zero $k^{\text {th }}$-moment, i.e. $\mu_{k}=\int_{-\infty}^{\infty} x^{k} \varphi(x) \mathrm{d} x \neq 0$, then $f$ has quasiasymptotic behavior of degree $k$ with respect to $L$.

Proof. As in the proof of Theorem 6, we obtain that (5.14) and (5.15) imply $f$ satisfies an asymptotic expansion of the form (5.3); furthermore, evaluating the asymptotic relation (5.3) at a $\phi$ with non-zero first $k$ moments and using the quasiasymptotic boundedness (5.15), we obtain (5.16) and 
$c(\varepsilon)=O(L(\varepsilon))$. Evaluating (5.16) at $\varphi$, we obtain that $c(\varepsilon) \sim B L(\varepsilon)$, for some constant $B$. This completes the proof.

\section{TAUBERIAN THEOREMS FOR QUASIASYMPTOTICS AT INFINITY.}

We now state the Tauberian theorems for asymptotic behavior at infinity, the proofs of Theorem 8 and Theorem 9 follow immediately from Proposition 3 and Corollary 2. The proofs of Corollaries 5-6 and Theorems 10-11 are analogous to those of Corollaries 3-4 and Theorems 6-7, and then we choose to omit them.

Theorem 8. Let $f \in \mathcal{S}^{\prime}(\mathbb{R})$ and $\alpha \notin \mathbb{N}$. Suppose the wavelet $\psi \in \mathcal{S}_{0}(\mathbb{R})$ admits a reconstruction wavelet. Necessary and sufficient conditions in terms of the wavelet transform for the existence of a polynomial $p$ such that $f-p$ has quasiasymptotic behavior at infinity of degree $\alpha$ with respect to a slowly varying function $L$ are the existence of the limits

$$
\lim _{\lambda \rightarrow \infty} \frac{1}{\lambda^{\alpha} L(\lambda)} \mathcal{W}_{\psi} f(\lambda b, \lambda a)=M_{b, a}, \quad \text { for each }(b, a) \in \mathbb{H},
$$

and the existence constants of $\gamma, \beta, M>0$ such that

$$
\frac{1}{\lambda^{\alpha} L(\lambda)}\left|\mathcal{W}_{\psi} f(\lambda b, \lambda a)\right|<M\left(a+\frac{1}{a}\right)^{\gamma}(1+|b|)^{\beta},
$$

for all $(b, a) \in \mathbb{H}$ and $\lambda \geq 1$. In such a case there is a homogeneous distribution $g$ of degree $\alpha$ such that $M_{b, a}=\mathcal{W}_{\psi} g(b, a),(b, a) \in \mathbb{H}$.

Theorem 9. Let $f \in \mathcal{S}^{\prime}(\mathbb{R})$ and $k \in \mathbb{N}$. Suppose the wavelet $\psi \in \mathcal{S}_{0}(\mathbb{R})$ admits a reconstruction wavelet. The conditions (6.1) and (6.2) with $\alpha=k$ are necessary and sufficient for the existence of a distribution of the form $g(x)=C_{-} x_{-}^{k}+C_{+} x_{+}^{k}+\beta x^{k} \log |x|$, a polynomial $p$, which is divisible by $x^{k+1}$, and an associate asymptotically homogeneous function $c$, satisfying (4.4), such that

$$
f(\lambda x)=p(\lambda x)+c(\lambda) x^{k}+\lambda^{k} L(\lambda) g(x)+o\left(\lambda^{k} L(\lambda)\right),
$$

as $\lambda \rightarrow \infty$ in $\mathcal{S}^{\prime}(\mathbb{R})$. Moreover, $M_{b, a}=\mathcal{W}_{\psi} g(b, a),(b, a) \in \mathbb{H}$.

Example 6. Let $f$ be an non-decreasing functions having tempered growth and being supported by $[0, \infty)$. Assume also that $\alpha \geq 0$. In this case, the conditions (6.1) and (6.2) are necessary and sufficient for $f$ to have asymptotic behavior

$$
f(x) \sim C x^{\alpha} L(x), \quad \text { for some } C .
$$

Indeed, the necessity follows at once. For the sufficiency, observe the support condition imposed to $f$ leads to the quasiasymptotic behavior

$$
f(\lambda x) \sim C \lambda^{\alpha} L(\lambda) x_{+}^{\alpha} \quad \text { as } \lambda \rightarrow \infty \text { in } \mathcal{S}^{\prime}(\mathbb{R}),
$$

for some $C$, which implies the asymptotic behavior in the ordinary sense (cf. [47, p. 124]). Of course, similar conclusions hold for non-negative measures and their primitives. 
Corollary 5. Let the hypothesis of Theorem 8 be satisfied. Set $n=[\alpha]$. Let $\varphi \in \mathcal{S}(\mathbb{R})$ be such that its moments $\mu_{j}:=\int_{-\infty}^{\infty} x^{j} \varphi(x) \mathrm{d} x \neq 0$, for $n<j$. The condition

$$
\langle f(\lambda x), \varphi(x)\rangle=O\left(\lambda^{k} L(\lambda)\right), \quad \lambda \rightarrow \infty,
$$

implies that $f$ has quasiasymptotic behavior of degree $\alpha$ with respect to $L$ at infinity.

Corollary 6. Let the hypothesis of Theorem 9 be satisfied. Let $\varphi \in \mathcal{S}(\mathbb{R})$ be such that its moments $\mu_{j}:=\int_{-\infty}^{\infty} x^{j} \varphi(x) \mathrm{d} x \neq 0$, for $k \leq j$. The condition

$$
\langle f(\lambda x), \varphi(x)\rangle \sim C \lambda^{k} L(\lambda), \quad \lambda \rightarrow \infty,
$$

for some constant $C$, implies that $f$ has quasiasymptotic behavior of degree $k$ with respect to $L$ at infinity.

Theorem 10. Let $f \in \mathcal{S}^{\prime}(\mathbb{R}), \alpha \notin \mathbb{N}$, and $L$ be a slowly varying function at infinity. Let $\psi \in \mathcal{S}_{0}(\mathbb{R})$ be a wavelet admitting a reconstruction wavelet. Suppose that the following limits exist:

$$
\lim _{\lambda \rightarrow \infty} \frac{1}{\lambda^{\alpha} L(\lambda)} \mathcal{W}_{\psi} f(\lambda b, \lambda a)=M_{b, a}, \quad \text { for each }(b, a) \in \mathbb{H} .
$$

Then, the Tauberian condition

$$
f(\lambda x)=O\left(\lambda^{\alpha} L(\lambda)\right) \quad \text { as } \lambda \rightarrow \infty \text { in } \mathcal{S}^{\prime}(\mathbb{R}),
$$

implies the existence of a homogeneous distribution $g$ of degree $\alpha$ such that $M_{b, a}=\mathcal{W}_{\psi} g(b, a)$ and

$$
f(\lambda x) \sim \lambda^{\alpha} L(\lambda) g(x) \quad \text { as } \lambda \rightarrow \infty \text { in } \mathcal{S}^{\prime}(\mathbb{R}) .
$$

Conversely, the quasiasymptotic behavior (6.8) implies (6.6) and (6.7).

Theorem 11. Let $f \in \mathcal{S}^{\prime}(\mathbb{R}), k \in \mathbb{N}$, and $L$ be a slowly varying function at infinity. Let $\psi \in \mathcal{S}_{0}(\mathbb{R})$ be a wavelet admitting a reconstruction wavelet. Suppose that the following limits exist:

$$
\lim _{\lambda \rightarrow \infty} \frac{1}{\lambda^{k} L(\lambda)} \mathcal{W}_{\psi} f(\lambda b, \lambda a)=M_{b, a}, \text { for each }(b, a) \in \mathbb{H} .
$$

Then, the Tauberian condition

$$
f(\lambda x)=O\left(\lambda^{\alpha} L(\lambda)\right) \quad \text { as } \lambda \rightarrow \infty \text { in } \mathcal{S}^{\prime}(\mathbb{R}),
$$

implies the existence of a distribution having the form $g(x)=C_{-} x_{-}^{k}+$ $C_{+} x_{+}^{k}+\beta x^{k} \log |x|$ and an associate asymptotically homogeneous function $c$, satisfying (4.4), such that $M_{b, a}=\mathcal{W}_{\psi} g(b, a)$ and

$$
f(\lambda x)=c(\lambda) \lambda^{k} x^{k}+\lambda^{k} L(\lambda) g(x)+o\left(\lambda^{k} L(\lambda)\right) \quad \text { as } \lambda \rightarrow \infty \text { in } \mathcal{S}^{\prime}(\mathbb{R}) .
$$

Moreover, $c(\lambda)=O(L(\lambda))$. Additionally, if there exists $\varphi \in \mathcal{S}^{\prime}(\mathbb{R})$ satisfying (6.5) and having non-zero $k^{t h}$-moment, i.e. $\mu_{k}=\int_{-\infty}^{\infty} x^{k} \varphi(x) \mathrm{d} x \neq 0$, then $f$ has quasiasymptotic behavior of degree $k$ with respect to $L$. 
Remark 3. We give several remarks about progressive and regressive distributions. In the case of a progressive wavelet $\psi$ we say that $\eta$ is a reconstruction wavelet for it if just the positive frequency part of (2.3) is satisfied. Analogously for regressive ones.

Suppose first that $f \in \mathcal{S}_{+}^{\prime}(\mathbb{R})$. Since only the positive frequency part of a wavelet is relevant for the wavelet transform of $f$, and any non-vanishing $\psi \in \mathcal{S}_{+}(\mathbb{R})$ is its own reconstruction wavelet, it is enough to assume in Propositions 3 and 4 that $\psi$ is an arbitrary non-zero element of $\mathcal{S}_{+}(\mathbb{R})$. Likewise, if $f \in \mathcal{S}_{-}^{\prime}(\mathbb{R})$, Propositions 3 and 4 hold for an arbitrary non-zero regressive $\psi \in \mathcal{S}_{-}(\mathbb{R})$.

Assume now that $f \in \mathcal{S}^{\prime}(\mathbb{R})$ is a progressive distribution, that is, supp $\hat{f} \subseteq$ $[0, \infty)$. Then, Theorems $3-11$ hold if $\psi$ is an arbitrary non-zero element of $\mathcal{S}_{+}(\mathbb{R})$. Similarly, for a regressive distribution, they hold for a arbitrary non-zero regressive $\psi \in \mathcal{S}_{-}(\mathbb{R})$.

\section{Appendix: Proof of Lemma 1}

Before going over the proof, we need some additional wavelet concepts [18].

The space $\mathcal{S}(\mathbb{H})$ of highly localized function over $\mathbb{H}$ is defined as those smooth functions on $\mathbb{H}$ such that

$$
\sup _{(b, a) \in \mathbb{H}}\left(a+\frac{1}{a}\right)^{m}\left(1+b^{2}\right)^{\frac{n}{2}}\left|\frac{\partial^{k+l} \Phi}{\partial a^{k} \partial b^{l}}(b, a)\right|<\infty,
$$

for all $m, n, k, l \in \mathbb{N}$. It is topologized in the obvious way. We will also consider its dual space, $\mathcal{S}^{\prime}(\mathbb{H})$. Any locally integrable function $F$ of "slow growth" on $\mathbb{H}$, that is,

$$
|F(b, a)| \leq C\left(1+b^{2}\right)^{\frac{l}{2}}\left(a+\frac{1}{a}\right)^{m}, \quad(b, a) \in \mathbb{H},
$$

for some $C>0$ and integers $m, l \in \mathbb{N}$, can be identified with an element of $\mathcal{S}^{\prime}(\mathbb{H})$. Our convention for identifying it with an element of $\mathcal{S}^{\prime}(\mathbb{H})$ is to keep using the notation $F \in \mathcal{S}^{\prime}(\mathbb{H})$ and the evaluation of $F$ at $\Phi \in \mathcal{S}(\mathbb{H})$ is given by

$$
\langle F(b, a), \Phi(b, a)\rangle=\int_{0}^{\infty} \int_{-\infty}^{\infty} F(b, a) \Phi(b, a) \frac{\mathrm{d} b \mathrm{~d} a}{a} .
$$

One defines the wavelet synthesis $\mathcal{M}_{\psi}: \mathcal{S}^{\prime}(\mathbb{H}) \rightarrow \mathcal{S}_{0}^{\prime}(\mathbb{R})$ by

$$
\left\langle\mathcal{M}_{\psi} F(x), \phi(x)\right\rangle=\left\langle F(b, a), \mathcal{W}_{\bar{\psi}} \phi(b, a)\right\rangle .
$$

Suppose $\eta$ is a reconstruction wavelet for $\psi$, we will make use of the projection operator of $\mathcal{S}^{\prime}(\mathbb{H})$ onto the image of the wavelet transform [18], it is given by the projector

$$
\frac{1}{c_{\psi, \eta}} \mathcal{W}_{\psi} \mathcal{M}_{\eta}
$$


If the distribution $F$ is a locally integrable distribution of slow growth on $\mathbb{H}$, it is possible to write the projection by the integral transform

$$
\frac{\mathcal{W}_{\psi} \mathcal{M}_{\eta} F(b, a)}{c_{\psi, \eta}}=\frac{1}{c_{\psi, \eta}} \int_{0}^{\infty} \int_{-\infty}^{\infty} \mathcal{W}_{\psi} \eta\left(\frac{b-b^{\prime}}{a^{\prime}}, \frac{a}{a^{\prime}}\right) F\left(b^{\prime}, a^{\prime}\right) \frac{\mathrm{d} b^{\prime} \mathrm{d} a^{\prime}}{\left(a^{\prime}\right)^{2}} .
$$

Proof of Lemma 1. Clearly (3.14) implies (3.13). Thus, we will prove that $(3.13) \Rightarrow(3.14)$. We assume that $x_{0}=0$ for simplicity. Let $\eta$ be a reconstruction wavelet for $\psi$.

Let $F=\chi_{I} \mathcal{W}_{\psi} f$ where $\chi_{I}$ is the characteristic function of the set $I=$ $|b| \leq 1,0<a \leq 1$. Let $G=\mathcal{W}_{\psi} f-F$. Consider $f_{0}=c_{\psi, \eta}^{-1} \mathcal{M}_{\eta} F$ and $h=c_{\psi, \eta}^{-1} \mathcal{M}_{\eta} G$. Notice that $\mathcal{W}_{\psi} f=\mathcal{W}_{\psi} h+\mathcal{W}_{\psi} f_{0}$, and hence $f=h+f_{0}$. The plan is to show that each $\mathcal{W}_{\psi} h$ and $\mathcal{W}_{\psi} f_{0}$ satisfy an estimate of type (3.14).

We will show first that $\mathcal{W}_{\psi} h(b, a)=o\left(a^{\infty}\right)$ uniformly for $b$ in a neighborhood of the origin as $a \rightarrow 0^{+}$. Let $\sigma$ be a positive real number. Find $n \in \mathbb{N}$ and $B>0$ such that

$$
|G(b, a)| \leq B\left(a+\frac{1}{a}\right)^{n}(1+|b|)^{n}
$$

and

$$
\left|\mathcal{W}_{\psi} \eta(b, a)\right| \leq B\left(a+\frac{1}{a}\right)^{-1-2 n-\sigma}(1+|b|)^{-2-n} .
$$

If $|b| \leq \frac{1}{2}$ and $a<1$, then, by (7.1),

$$
\begin{aligned}
& \left|c_{\psi, \eta} \mathcal{W}_{\psi} h(b, a)\right|=\left|\int_{1}^{\infty} \int_{\left|b^{\prime}\right| \geq 1} \mathcal{W}_{\psi} \eta\left(\frac{b-b^{\prime}}{a^{\prime}}, \frac{a}{a^{\prime}}\right) G\left(b^{\prime}, a^{\prime}\right) \frac{\mathrm{d} b^{\prime} \mathrm{d} a^{\prime}}{\left(a^{\prime}\right)^{2}}\right| \\
& \leq 4^{n} B^{2} \int_{1}^{\infty} \int_{\left|b^{\prime}\right| \geq 1}\left|b^{\prime}\right|^{n}\left(a^{\prime}\right)^{n}\left(\frac{a^{\prime}}{\left|b-b^{\prime}\right|}\right)^{2+n}\left(\frac{a}{a^{\prime}}\right)^{1+2 n+\sigma} \frac{\mathrm{d} b^{\prime} \mathrm{d} a^{\prime}}{\left(a^{\prime}\right)^{2}} \\
& \leq a^{1+2 n+\sigma} 4^{n} B^{2}\left(\int_{\left|b^{\prime}\right| \geq 1} \frac{\left|b^{\prime}\right|^{n} \mathrm{~d} b^{\prime}}{\left(\left|b^{\prime}\right|-\frac{1}{2}\right)^{n+2}}\right)\left(\int_{1}^{\infty} \frac{\mathrm{d} a^{\prime}}{\left(a^{\prime}\right)^{\sigma+1}}\right) \\
& =o\left(a^{\sigma}\right) .
\end{aligned}
$$

We use the characterization of the singular support of distributions given in [18, Thm. 27.0.2], and conclude that $h$ is $C^{\infty}$ in $(-1 / 2,1 / 2)$. In particular, $h(\varepsilon x)=o\left(\varepsilon^{\infty}\right)$, and so the wavelet characterization of bounded sets in $\mathcal{S}_{0}^{\prime}(\mathbb{R})$ [18, Thm. 28.0.1] implies that $\mathcal{W}_{\psi} h$ satisfies an estimate of the form (3.14).

Let us now show that $\mathcal{W}_{\psi} f_{0}$ satisfies an estimate of the form (3.14). Observe that (3.13) implies

$$
\frac{a^{m}}{\varepsilon^{\alpha} L(\varepsilon)}\left|\mathcal{W}_{\psi} f(\varepsilon b, \varepsilon a)\right|<M_{1}, \text { for all } a^{2}+b^{2}=1, a>0,0<\varepsilon \leq \varepsilon_{0},
$$

for some $M_{1}>0$ and $\varepsilon_{0}$. After rescaling, i.e., replacing if necessary $f_{0}(x)$ by $f_{0}\left(\varepsilon_{0}^{-1} \sqrt{2} x\right)$, we may assume $\varepsilon_{0}=\sqrt{2}$. Let $a^{\prime} \in\left(0, \varepsilon^{-1}\right)$ and $b^{\prime} \in$ 
$\left(-\varepsilon^{-1}, \varepsilon^{-1}\right)$. Then we have for $\varepsilon<1$ that $\varepsilon \sqrt{\left(a^{\prime}\right)^{2}+\left(b^{\prime}\right)^{2}} \leq \sqrt{2}$. So, if we replace $a, b$ and $\varepsilon$ by $a^{\prime} / \sqrt{\left(a^{\prime}\right)^{2}+\left(b^{\prime}\right)^{2}}, b^{\prime} / \sqrt{\left(a^{\prime}\right)^{2}+\left(b^{\prime}\right)^{2}}$ and $\varepsilon \sqrt{\left(a^{\prime}\right)^{2}+\left(b^{\prime}\right)^{2}}$, we obtain that for $a^{\prime} \in\left(0, \varepsilon^{-1}\right), b^{\prime} \in\left(-\varepsilon^{-1}, \varepsilon^{-1}\right)$

$$
\frac{\left(a^{\prime}\right)^{m}\left|\mathcal{W}_{\psi} f\left(\varepsilon b^{\prime}, \varepsilon a^{\prime}\right)\right|}{\varepsilon^{\alpha}\left(\sqrt{\left(a^{\prime}\right)^{2}+\left(b^{\prime}\right)^{2}}\right)^{m+\alpha} L\left(\varepsilon \sqrt{\left(a^{\prime}\right)^{2}+\left(b^{\prime}\right)^{2}}\right)}<M_{1}, \quad 0<\varepsilon \leq 1 .
$$

In addition, we can assume that $\alpha+m \geq 1$. We also need to make a technical assumption over $L$ which can be always made since only the values of $L$ near 0 matter for our considerations; indeed we can assume ([46, Section 2], [1, p.25]) that there exists a constant $M_{2}>0$ such that

$$
\frac{L(\varepsilon x)}{L(\varepsilon)} \leq M_{2} \max \left\{x, x^{-1}\right\} \leq M_{2} \frac{1+x^{2}}{x}, \text { for all } \varepsilon, x>0
$$

Let

$$
\beta=\alpha+m+3, \quad \gamma=\max \{m+2, \alpha+\beta+1\} .
$$

Find now a constant $M_{3}>0$ such that

$$
\left|\mathcal{W}_{\psi} \eta(b, a)\right| \leq M_{3}\left(a+\frac{1}{a}\right)^{-\gamma}(1+|b|)^{-\beta}
$$

In the following, we will also make repeated use of the elementary inequality

$$
1+|x+y| \leq(1+|x|)(1+|y|) .
$$


Then for $0<\varepsilon \leq 1$, we have from (7.2), (7.3) and (7.6) that

$$
\begin{aligned}
&\left|c_{\psi, \eta} \mathcal{W}_{\psi} f_{0}(\varepsilon b, \varepsilon a)\right|=\left|\int_{0}^{\infty} \int_{-\infty}^{\infty} \mathcal{W}_{\psi} \eta\left(\frac{\varepsilon b-b^{\prime}}{a^{\prime}}, \frac{\varepsilon a}{a^{\prime}}\right) F\left(b^{\prime}, a^{\prime}\right) \frac{\mathrm{d} b^{\prime} \mathrm{d} a^{\prime}}{\left(a^{\prime}\right)^{2}}\right| \\
&=\left|\int_{0}^{\varepsilon^{-1}} \int_{-\varepsilon^{-1}}^{\varepsilon^{-1}} \mathcal{W}_{\psi} \eta\left(\frac{b-b^{\prime}}{a^{\prime}}, \frac{a}{a^{\prime}}\right) \mathcal{W}_{\psi} f\left(\varepsilon b^{\prime}, \varepsilon a^{\prime}\right) \frac{\mathrm{d} b^{\prime} \mathrm{d} a^{\prime}}{\left(a^{\prime}\right)^{2}}\right| \\
& \leq M_{1} \varepsilon^{\alpha} \int_{0}^{\varepsilon^{-1}} \int_{-\varepsilon^{-1}}^{\varepsilon^{-1}}\left(\sqrt{\left(a^{\prime}\right)^{2}+\left(b^{\prime}\right)^{2}}\right)^{\alpha+m} \\
& \leq L\left(\varepsilon \sqrt{\left(a^{\prime}\right)^{2}+\left(b^{\prime}\right)^{2}}\right)\left|\mathcal{W}_{\psi} \eta\left(\frac{b-b^{\prime}}{a^{\prime}}, \frac{a}{a^{\prime}}\right)\right| \frac{\mathrm{d} b^{\prime} \mathrm{d} a^{\prime}}{\left(a^{\prime}\right)^{m+2}} \\
& \leq M_{1} M_{2} \varepsilon^{\alpha} L(\varepsilon) \int_{0}^{\varepsilon^{-1}} \int_{-\varepsilon^{-1}}^{\varepsilon^{-1}}\left(\sqrt{\left(a^{\prime}\right)^{2}+\left(b^{\prime}\right)^{2}}\right)^{\alpha+m-1} \\
& \quad\left(1+\left(a^{\prime}\right)^{2}+\left(b^{\prime}\right)^{2}\right)\left|\mathcal{W}_{\psi} \eta\left(\frac{b-b^{\prime}}{a^{\prime}}, \frac{a}{a^{\prime}}\right)\right| \frac{\mathrm{d} b^{\prime} \mathrm{d} a^{\prime}}{\left(a^{\prime}\right)^{m+2}} \\
& \leq M_{1} M_{2} \varepsilon^{\alpha} L(\varepsilon) \int_{0}^{\varepsilon^{-1}} \int_{-\varepsilon^{-1}}^{\varepsilon^{-1}}\left(a^{\prime}+\left|b^{\prime}\right|\right)^{\alpha+m-1}\left(1+a^{\prime}\right)^{2}\left(1+\left|b^{\prime}\right|\right)^{2} \\
&\left|\mathcal{W}_{\psi} \eta\left(\frac{b-b^{\prime}}{a^{\prime}}, \frac{a}{a^{\prime}}\right)\right| \frac{\mathrm{d} b^{\prime} \mathrm{d} a^{\prime}}{\left(a^{\prime}\right)^{m+2}} \\
& \leq M_{1} M_{2} \varepsilon^{\alpha} L(\varepsilon)\left(4 I_{1}+4 I_{2}+2^{\alpha+m+1} I_{3}\right),
\end{aligned}
$$

where

$$
\begin{aligned}
& I_{1}=\int_{0}^{1} \int_{\left|b-b^{\prime}\right| \leq 1}\left(1+\left|b^{\prime}\right|\right)^{\alpha+m+1}\left|\mathcal{W}_{\psi} \eta\left(\frac{b-b^{\prime}}{a^{\prime}}, \frac{a}{a^{\prime}}\right)\right| \frac{\mathrm{d} b^{\prime} \mathrm{d} a^{\prime}}{\left(a^{\prime}\right)^{m+2}}, \\
& I_{2}=\int_{0}^{1} \int_{1 \leq\left|b-b^{\prime}\right|}\left(1+\left|b^{\prime}\right|\right)^{\alpha+m+1}\left|\mathcal{W}_{\psi} \eta\left(\frac{b-b^{\prime}}{a^{\prime}}, \frac{a}{a^{\prime}}\right)\right| \frac{\mathrm{d} b^{\prime} \mathrm{d} a^{\prime}}{\left(a^{\prime}\right)^{m+2}}, \\
& I_{3}=\int_{1}^{\infty} \int_{-\infty}^{\infty}\left(a^{\prime}\right)^{\alpha-1}\left(1+\left|b^{\prime}\right|\right)^{\alpha+m+1}\left|\mathcal{W}_{\psi} \eta\left(\frac{b-b^{\prime}}{a^{\prime}}, \frac{a}{a^{\prime}}\right)\right| \mathrm{d} b^{\prime} \mathrm{d} a^{\prime} .
\end{aligned}
$$

To estimate the last three integrals, we make use of (7.4), (7.5) and the elementary inequality (7.6). We have

$$
\begin{aligned}
I_{1} & \leq M_{3} \int_{0}^{1} \int_{\left|b^{\prime}\right| \leq 1+|b|}\left(1+\left|b^{\prime}\right|\right)^{\alpha+m+1}\left(\frac{a^{\prime}}{a}\right)^{\gamma} \frac{\mathrm{d} b^{\prime} \mathrm{d} a^{\prime}}{\left(a^{\prime}\right)^{m+2}} \\
& \leq 2 M_{3}\left(\frac{1}{a}\right)^{\gamma}(1+|b|)(2+|b|)^{\alpha+m+1} \int_{0}^{1}\left(a^{\prime}\right)^{\gamma-m-2} \mathrm{~d} a^{\prime} \\
& <2^{\alpha+m+2} M_{3}\left(a+\frac{1}{a}\right)^{\gamma}(1+|b|)^{\beta}
\end{aligned}
$$


for $I_{2}$,

$$
\begin{aligned}
I_{2} & \leq M_{3} \int_{0}^{1} \int_{1<\left|b-b^{\prime}\right|}\left(1+\left|b^{\prime}\right|\right)^{\alpha+m+1}\left(\frac{a^{\prime}}{a}\right)^{\gamma}\left(\frac{a^{\prime}}{a^{\prime}+\left|b-b^{\prime}\right|}\right)^{\beta} \frac{\mathrm{d} b^{\prime} \mathrm{d} a^{\prime}}{\left(a^{\prime}\right)^{m+2}} \\
& \leq M_{3}\left(a+\frac{1}{a}\right)^{\gamma}\left(\int_{0}^{1}\left(a^{\prime}\right)^{\gamma+\beta-m-2} \mathrm{~d} a^{\prime}\right)\left(\int_{1<\left|b-b^{\prime}\right|} \frac{\left(1+\left|b^{\prime}\right|\right)^{\alpha+m+1}}{\left|b-b^{\prime}\right|^{\beta}} \mathrm{d} b^{\prime}\right) \\
& \leq M_{3}\left(a+\frac{1}{a}\right)^{\gamma} \int_{1<\left|b^{\prime}\right|} \frac{\left(1+\left|b^{\prime}\right|+|b|\right)^{\alpha+m+1}}{\left|b^{\prime}\right|^{\beta}} \mathrm{d} b^{\prime} \\
& \leq 2 M_{3}\left(a+\frac{1}{a}\right)^{\gamma}(1+|b|)^{\alpha+m+1} \int_{1}^{\infty} \frac{\left(1+b^{\prime}\right)^{\alpha+m+1}}{\left(b^{\prime}\right)^{\beta}} \mathrm{d} b^{\prime} \\
& \leq 2^{\alpha+m+2} M_{3}\left(a+\frac{1}{a}\right)^{\gamma}(1+|b|)^{\beta} ;
\end{aligned}
$$

and finally $I_{3}$,

$$
\begin{aligned}
I_{3} & \leq M_{3} a^{\gamma} \int_{1}^{\infty} \int_{-\infty}^{\infty} \frac{\left(a^{\prime}\right)^{\alpha+\beta-\gamma-1}\left(1+\left|b^{\prime}\right|\right)^{\alpha+m+1}}{\left(a^{\prime}+\left|b-b^{\prime}\right|\right)^{\beta}} \mathrm{d} b^{\prime} \mathrm{d} a^{\prime} \\
& \leq M_{3}\left(a+\frac{1}{a}\right)^{\gamma}\left(\int_{-\infty}^{\infty} \frac{\left(1+\left|b^{\prime}\right|\right)^{\alpha+m+1}}{\left(1+\left|b-b^{\prime}\right|\right)^{\beta}} \mathrm{d} b^{\prime}\right)\left(\int_{1}^{\infty} \frac{\mathrm{d} a^{\prime}}{\left(a^{\prime}\right)^{\gamma+1-\beta-\alpha}}\right) \\
& \leq M_{3}\left(a+\frac{1}{a}\right)^{\gamma}(1+|b|)^{\beta} \int_{-\infty}^{\infty} \frac{\mathrm{d} b^{\prime}}{\left(1+\left|b^{\prime}\right|\right)^{2}} \\
& \leq 2 M_{3}\left(a+\frac{1}{a}\right)^{\gamma}(1+|b|)^{\beta} .
\end{aligned}
$$

Hence (3.14) is satisfied with $M=2^{\alpha+m+6} M_{1} M_{2} M_{3}$.

\section{REFERENCES}

[1] N. H. Bingham, C. M. Goldie, J. L. Teugels, Regular variation, Encyclopedia of Mathematics and its Applications 27, Cambridge Univ. Press, Cambridge, 1989.

[2] N. N. Bogoliubov, D.V. Shirkov, Introduction to the Theory of Quantized Fields, Wiley, New York, 1959.

[3] J. M. Bony, Second microlocalization and propagation of singularities for semilinear hyperbolic equations, Tanaguchi Symp. HERT. Katata (1984), 11-49.

[4] O. Christensen, An introduction to frames and Riesz bases, Applied and Numerical Harmonic Analysis, Birkhäuser, Boston, 2003.

[5] J. B. Conway, Functions of One Complex Variable II, Springer, New York, 1995.

[6] I. Daubechies, Ten Lectures on Wavelets, SIAM, Phyladelphia, 1992.

[7] Y. N. Drozhzhinov, B. I. Zavialov, Tauberian-type theorems for a generalized multiplicative convolution, Izv. Math. 64 (2000), 35-92.

[8] Y. N. Drozhzhinov, B. I. Zavialov, Tauberian theorems for generalized functions with values in Banach spaces, Izv. Math. 66 (2002), 701-769.

[9] Y. N. Drozhzhinov, B. I. Zavialov, Asymptotically homogeneous generalized functions, Izv. Nats. Akad. Nauk Armenii Mat. 41 (2006), 23-32. 
[10] Y. N. Drozhzhinov, B. I. Zavialov, Asymptotically quasihomogeneous generalized functions, Dokl. Akad. Nauk 421 (2008), 157-161.

[11] A. L. Durán, R. Estrada, Strong moment problems for rapidly decreasing smooth functions, Proc. Amer. Math. Soc. 120 (1994), 529-534.

[12] R. Estrada, Characterization of the Fourier series of distributions having a value at a point, Proc. Amer. Math. Soc. 124 (1996), 1205-1212.

[13] R. Estrada, The nonexistence of regularization operators, J. Math. Anal. Appl. 286 (2003), 1-10.

[14] R. Estrada, R. P. Kanwal, Singular Integral Equations, Birkhäuser, Boston, 2000.

[15] R. Estrada, R. P. Kanwal, A distributional approach to Asymptotics. Theory and Applications, second edition, Birkhäuser, Boston, 2002.

[16] H. G. Feichtinger, K. Gröchenig, Gabor frames and time-frequency analysis of distributions, J. Funct. Anal. 146 (1997), 464-495.

[17] K. Gröchenig, Foundation of Time-Frequency Analysis, Birkhäuser, Boston, 2001.

[18] M. Holschneider, Wavelets. An analysis tool, The Clarendon Press, Oxford Univ. Press, New York, 1995.

[19] M. Holschneider, Ph. Tchamitchian, Pointwise analysis of Riemann's "nondifferentiable" function, Invent. Math. 105 (1991), 157-175.

[20] L. Hörmander, Lectures on Nonlinear Hyperbolic Differential Equations, Mathematiques \& Applications 26, Springer, Berlin, 1997.

[21] S. Jaffard, The spectrum of singularities of Riemann's function, Rev. Mat. Iberoamericana 12 (1996), 441-460

[22] S. Jaffard, Wavelet expansions, function spaces and multifractal analysis, Twentieth century harmonic analysis - a celebration (Il Ciocco, 2000), 127-144, NATO Sci. Ser. II Math. Phys. Chem., 33, Kluwer Acad. Publ., Dordrecht, 2001.

[23] S. Jaffard, Y. Meyer, Wavelet Methods for Pointwise Regularity and Local Oscillations of Functions, Memoirs of the American Mathematical Society, vol.123, No 587, 1996.

[24] S. Lazzarini, J. M. Gracia-Bondía, Improved Epstein-Glaser renormalization. II. Lorentz invariant framework, J. Math. Phys. 44 (2003), 3863-3875.

[25] J. E. Littlewood, The converse of Abel's theorem on power series, Proc. London Math. Soc. 9 (1911), 434-448.

[26] S. Łojasiewicz, Sur la valeur et la limite d'une distribution en un point, Studia Math. 16 (1957), 1-36.

[27] Y. Meyer, Wavelets and Operators, Cambridge Univ. Press, Cambridge, 1992.

[28] Y. Meyer, Wavelets, vibrations and scalings, CRM Monograph series 9, American Mathematical Society, Providence, 1998.

[29] S. Pilipović, Quasiasymptotics and $S$-asymptotics in $\mathcal{S}^{\prime}$ and $\mathcal{D}^{\prime}$, Publ. Inst. Math. (Beograd) 72 (1995), 13-20.

[30] S. Pilipović, B. Stanković, A. Takači, Asymptotic Behaviour and Stieltjes Transformation of Distributions, Teubner-Texte zuer Mathematik, Leipzig, 1990.

[31] S. Pilipović, N. Teofanov, Multiresolution expansion, approximation order and quasiasymptotic behavior of tempered distributions, J. Math. Anal. Appl. 331 (2007), 455471.

[32] K. Saneva, A. Bučkovska, Asymptotic expansion of distributional wavelet transform, Integral Transforms Spec. Funct. 17 (2006), 85-91.

[33] K. Saneva, A. Bučkovska, Tauberian theorems for distributional wavelet transform, Integral Transforms Spec. Funct. 18 (2007), 359-368.

[34] L. Schwartz, Théorie des Distributions, Hermann, Paris, 1966.

[35] S. Seuret, J. L. Véhel, The local Hölder function of a continuous function, Appl, Comput Harmonic Anal. 13 (2002), 263-276.

[36] S. Seuret, J. L. Véhel, A Time Domain Characterization of 2-Microlocal Spaces, J. Fourier Anal. Appl., 9 (2003), 473-495. 
[37] V. M. Shelkovich, Associated and quasi associated homogeneous distributions (generalized functions), J. Math. Anal. Appl. 338 (2008), 48-70.

[38] B. K. Sohn, D. H. Pahk, Pointwise convergence of wavelet expansion of $\mathcal{K}_{r}^{M^{\prime}}(\mathbb{R})$, Bull. Korean Math. Soc. 38 (2001), 81-91.

[39] E. M. Stein, Singular integrals and differentiability properties of functions, Princeton Univ. Press, Princeton, New Jersey, 1970.

[40] F. Trèves, Topological Vector Spaces, Distributions and Kernel, Academic Press, New York, 1967.

[41] H. Triebel, Wavelet frames for distributions; local and pointwise regularity, Studia Math. 154 (2003), 59-88.

[42] J. Vindas, Structural Theorems for Quasiasymptotics of Distributions at Infinity, Publ. Inst. Math. (Beograd) (N.S.) 84(98)(2008), 159-174.

[43] J. Vindas, The structure of quasiasymptotics of Schwartz distributions, Linear and non-linear theory of generalized functions and its applications, pp. 297-314, Banach Center Publ. 88, Polish Acad. Sc. Inst. Math., Warsaw, 2010.

[44] J. Vindas, E. Estrada, Distributional Point Values and Convergence of Fourier Series and Integrals, J. Fourier Anal. Appl. 13 (2007), 551-576.

[45] J. Vindas, R. Estrada, On the jump behavior of distributions and logarithmic averages, J. Math. Anal. Appl. 347 (2008), 597-606.

[46] J. Vindas, S. Pilipović, Structural theorems for quasiasymptotics of distributions at the origin, Math. Nachr. 282 (2009), 1584-1599.

[47] V. S. Vladimirov, Y. N. Drozhzhinov, B. I. Zavialov, Tauberian theorems for generalized functions, Kluwer Acad. Publ., Dordrecht, 1988.

[48] V. S. Vladimirov, Y. N. Drozhzhinov, B. I. Zavialov, Tauberian theorems for generalized functions in a scale of regularly varying functions and functionals, dedicated to Jovan Karamata, Publ. Inst. Math. (Beograd) (N.S.) 71 (2002), 123-132 (in Russian).

[49] V. S. Vladimirov, B. I. Zavialov, On the Tauberian Theorems in Quantum Field Theory, Theoret. Mat. Fiz. 40 (1979), 155-178.

[50] V. S. Vladimirov, B. I. Zavialov, Tauberian Theorems in Quantum Field Theory, in: Current Problems in Mathematics, vol.15 (in Russian), pp.95-130, 228, Akad. Nauk SSSR, Vsesoyuz. Inst. Nauchn. i Tekhn. Informatsii, Moscow, 1980.

[51] P. Wagner, On the quasi-asymptotic expansion of the casual fundamental solution of hyperbolic operators and systems, Z. Anal. Anwendungen 10 (1991), 159-167.

[52] G. Walter, Pointwise convergence of wavelet expansions, J. Approx. Theory 80 (1995), 108-118.

[53] G. Walter, X. Shen, Wavelets and other orthogonal systems, second edition, Studies in Advanced Mathematics, Chapman \& Hall/CRC, Boca Raton, 2001.

[54] B. I. Zavialov, Aling of electromagnetic form factors and the behavior of their Fourier transforms in the neighborhood of the light cone, Teoret. Mat. Fiz. 17 (1973), 178-188.

Department of Mathematics, Ghent University, Krijgslaan 281 Gebouw S22, B 9000 Gent, Belgium

E-mail address: jvindas@cage.ugent.be

University of Novi Sad, Department of Mathematics and Informatics, TrG Dositeja Obradovića 4, 21000 Novi Sad, Serbia

E-mail address: pilipovic@dmi.uns.ac.rs

University of Novi Sad, Faculty of Technology, Bul. Cara Lazara 1, 21000

Novi Sad, Serbia

E-mail address: drakic@tf.uns.ac.rs 